\title{
One-loop QCD thermodynamics in a strong homogeneous and static magnetic field
}

\author{
Shubhalaxmi Rath and Binoy Krishna Patra \\ Department of Physics, Indian Institute of Technology Roorkee, \\ Roorkee 247667, India \\ E-mail: srath.dph2015@iitr.ac.in, binoyfph@iitr.ac.in
}

ABSTRACT: We have studied how the equation of state of thermal QCD with two light flavors is modified in a strong magnetic field. We calculate the thermodynamic observables of hot QCD matter up to one-loop, where the magnetic field affects mainly the quark contribution and the gluon part is largely unaffected except for the softening of the screening mass. We have first calculated the pressure of a thermal QCD medium in a strong magnetic field, where the pressure at fixed temperature increases with the magnetic field faster than the increase with the temperature at constant magnetic field. This can be understood from the dominant scale of thermal medium in the strong magnetic field, being the magnetic field, in the same way that the temperature dominates in a thermal medium in the absence of magnetic field. Thus although the presence of a strong magnetic field makes the pressure of hot QCD medium larger, the dependence of pressure on the temperature becomes less steep. Consistent with the above observations, the entropy density is found to decrease with the temperature in the presence of a strong magnetic field which is again consistent with the fact that the strong magnetic field restricts the dynamics of quarks to two dimensions, hence the phase space becomes squeezed resulting in the reduction of number of microstates. Moreover the energy density is seen to decrease and the speed of sound of thermal QCD medium increases in the presence of a strong magnetic field. These findings could have phenomenological implications in heavy ion collisions because the expansion dynamics of the medium produced in non-central ultra-relativistic heavy ion collisions is effectively controlled by both the energy density and the speed of sound.

KeYwords: Perturbative QCD, Quark-Gluon Plasma

ARXIV EPRINT: 1707.02890 


\section{Contents}

1 Introduction 1

2 The effective quark propagator in a strongly-magnetized hot QCD medium

2.1 Vacuum propagators in a strong magnetic field

2.2 Thermalization of propagators in a strong magnetic field 6

2.2.1 Quark propagator 6

$\begin{array}{ll}\text { 2.2.2 Gluon propagator } & 7\end{array}$

$\begin{array}{lll}2.3 & \text { One-loop quark self-energy in a strongly magnetized medium } & 7\end{array}$

2.3.1 Vacuum contribution $\quad 8$

$\begin{array}{lll}2.3 .2 & \text { Medium contribution } & 10\end{array}$

3 The effective gluon propagator in a strongly-magnetized hot QCD medium

$\begin{array}{lll}3.1 & \text { One-loop gluon self-energy in a hot QCD medium } & 13\end{array}$

$\begin{array}{lll}3.2 & \text { Screening mass in a strong magnetic field } & 15\end{array}$

4 Thermodynamic observables in the strong magnetic field 19

$\begin{array}{lll}4.1 & \text { Free energy and pressure } & 19\end{array}$

$\begin{array}{lll}4.1 .1 & \text { Quark contribution } & 19\end{array}$

$\begin{array}{lll}4.1 .2 & \text { Gluon contribution } & 21\end{array}$

4.1.3 Total pressure $\quad 23$

4.2 Entropy density 24

$\begin{array}{lll}4.3 & \text { Energy density } & 27\end{array}$

4.4 Speed of sound 28

5 Conclusions $\quad 30$

\section{Introduction}

To unravel the early history of the universe, it is of paramount importance to understand how a strongly-interacting matter behaves under the extreme conditions. Since more than a decade it has become a reality to create a matter of deconfined quarks and gluons (dubbed as QGP), similar to the matter in the early universe around one microsecond after the big bang, in the laboratory by the head-on collision between two heavy ions ultra-relativistically at RHIC, BNL and at LHC, CERN etc. However, only a few events are truly head-on, indeed most events occur at a finite impact parameter or centrality. As a result, the two highly charged ions in non-central events may produce extremely large magnetic fields $\sim m_{\pi}^{2}$ 
$\left(\simeq 10^{18}\right.$ Gauss $)$ at RHIC and $\sim 15 m_{\pi}^{2}$ at LHC $[1,2]$. Naive classical estimates of the lifetime of these magnetic fields indicate that they only exist for a small fraction of the lifetime of QGP $[3,4]$. However, depending on the transport coefficients of the medium produced, the magnetic field may be near to its maximum strength and it may be stationary [5-8] in its lifetime. Moreover the magnetic field may be assumed uniform because even though the spatial distribution of the magnetic field is globally inhomogeneous, but in the central region of the overlapping nuclei, the magnetic field in the transverse plane varies very smoothly, which is noticed in the hadron-string simulations [9] for $\mathrm{Au}-\mathrm{Au}$ collisions at $\sqrt{s_{N N}}=200 \mathrm{GeV}$ with an impact parameter, $b=10 \mathrm{fm}$. Therefore, a large number of QCD related phenomena are investigated in the strong and homogeneous magnetic field, such as the chiral magnetic effect related to the generation of electric current parallel to the magnetic field due to the difference in number of right and left-handed quarks [10-12], the axial magnetic effect due to the flow of energy by the axial magnetic field [13, 14], the chiral vortical effect due to an effective magnetic field in the rotating QGP $[15,16]$, the magnetic catalysis and the inverse magnetic catalysis at finite temperature arising due to the breaking and the restoration of the chiral symmetry [17-21], the thermodynamic properties [22-24], the refractive indices and decay constant $[25,26]$ of mesons in a hot magnetized medium, the conformal anomaly and the production of soft photons $[27,28]$ at RHIC and LHC, the dispersion relation in a magnetized thermal QED [29], the synchrotron radiation [30], the dilepton production from both the weakly [31-34] and the strongly [35] coupled plasma etc.

A variety of studies on the effects of strong magnetic fields particularly on the QCD thermodynamics have been studied by the possibility to achieve the strong magnetic fields at RHIC and LHC. Since the magnetic field breaks the translational invariance of space, so the pressure becomes anisotropic arising due to the difference between the pressures, which are transverse and longitudinal to the direction of background magnetic field, which is illustrated in an ensemble of spin one-half particles [24]. Recently the lattice QCD simulations [36] have explored the effects of background magnetic fields on the equation of state (EoS) by calculating the thermodynamic observables, namely the transverse and longitudinal pressures, the magnetization, the energy density, the entropy density etc. and have inferred that the transition temperature is reduced by the presence of a magnetic field [37-42]. Similarly the effects of strong magnetic fields on the phase structures of the hadronic matter have been reviewed in [23] through the low-energy effective theories, where the thermodynamic quantities are also found to increase with the magnetic field [43]. Thus the works referred above show that the EoS for the strongly-interacting matter have been modified by the presence of a magnetic field and this motivates us to study the EoS for QCD perturbatively in a strong magnetic field (SMF).

For a thermal medium, the free energy in non-abelian gauge theories have been analytically computed up to $\mathcal{O}\left(g^{4}\right)[44,45], \mathcal{O}\left(g^{5}\right)[46], \mathcal{O}\left(g^{6} \ln (1 / g)\right)$ [47] (where $g$ is the coupling constant). However, the thermodynamic observables obtained by the addition of successive higher-order terms in the weak-coupling expansion oscillate haphazardly, as a result they have a small radius of convergence. Hence a systematic reorganization of the perturbative expansions becomes essential for more convergent successive approximations 
to observables. In this process, various renormalization scales and effective field theory methods have emerged, such as the hard thermal loop (HTL) perturbation theory, where the resummation is achieved by the dominant parts of one-loop self-energies at various momentum scales, viz. $T, g T$ etc. [48-52].

However, in the presence of a magnetic field, the thermal medium possesses additional scales related to the magnetic field and depending on the strength of magnetic field compared to the temperature and the masses of quarks, the QCD thermodynamics is usually studied in two scenarios: in the weak magnetic field limit $\left(T^{2} \gg\left|q_{f} B\right|, T^{2} \gg m_{f}^{2}\right.$, where $\left|q_{f}\right|$ and $m_{f}$ are the absolute charge and the mass of the flavor, $f$, respectively), the temperature remains the dominant scale of the medium, so the HTL perturbation theory is still used to express the resumed quark propagator in powers of $\left|q_{f} B\right|$ [53]. On the other hand, in the strong magnetic field limit $\left(\left|q_{f} B\right| \gg T^{2},\left|q_{f} B\right| \gg m_{f}^{2}\right)$, we calculate the thermodynamic observables by replacing the upper limit of the loop momenta by the magnetic field $(\sqrt{e B})$, because the dominant scale in SMF limit is the magnetic field, in the same way that the temperature acts as the dominant scale in the absence of a magnetic field.

In the presence of a magnetic field, the quark momentum $\mathbf{p}$ is separated into components, which are transverse and longitudinal to the direction of magnetic field (say, $z$-direction), hence the dispersion relation for the quark is modified quantum mechanically into

$$
E_{n}\left(p_{z}\right)=\sqrt{p_{z}^{2}+m_{f}^{2}+2 n\left|q_{f} B\right|},
$$

where $n=0,1,2, \cdots$ are the quantum numbers to specify the Landau levels. In the strong magnetic field, the quarks are rarely excited thermally to the higher Landau levels, so only the lowest Landau levels (LLL) $(n=0)$ are populated. Thus the dynamics of quarks are effectively restricted to $(1+1)$ dimensions. In addition, the quark propagator is also modified in the presence of a magnetic field, which was first derived in the coordinate-space by Schwinger using the proper-time method [54] and later by Tsai [55] in the momentum-space. With the above mentioned modifications, we wish to calculate the one-loop contribution to the thermodynamic observables of a hot QCD matter in the SMF limit.

Our work proceeds in the following way. In section 2, we have derived the effective quark propagator in a thermal QCD medium in the strong magnetic field. So we have first revisited the vacuum quark and gluon propagators in the SMF limit and then thermalize them in the real-time formalism, in subsections 2.1 and 2.2, respectively. Using these propagators we compute the one-loop quark self-energy in section 2.3 in a strongly magnetized thermal medium. Next we have concentrated on the effective gluon propagator in the similar environment in section 3. In section 3.1, we have first revisited the covariant decomposition of the gluon self-energy in a thermal medium, which, in turn, leads to the decomposition of the effective gluon propagator. The effect of the magnetic field on the effective propagator enters through the screening (Debye) mass, which is calculated up to one-loop in section 3.2 for both the massless and the physical light quark masses. Having thus obtained the effective propagators for both quarks and gluons, we have then calculated the quark and gluon contributions to the free energy in section 4, from which we have computed all the thermodynamic observables of the hot QCD matter in the SMF limit. Finally we concluded in section 5 . 


\section{The effective quark propagator in a strongly-magnetized hot QCD medium}

The effective quark propagator in a strongly-magnetized hot QCD medium is obtained from the Schwinger-Dyson equation:

$$
S^{-1}(P)=S_{0}^{-1}(P)-\Sigma(P),
$$

where $S_{0}(P)$ and $\Sigma(P)$ are the free quark propagator and the quark self-energy in the medium, respectively.

As mentioned earlier, the strong magnetic field affects the quark propagator via the projection operator and the dispersion relation, which will, in turn affect the quark selfenergy. In addition, the QCD coupling runs exclusively with the magnetic field in SMF limit. Due to the presence of a magnetic field, the coupling is split into terms, which depend on the longitudinal and transverse components of the momentum [56]. For the magnetic field in the $z$-direction $(\mathbf{B}=B \hat{z})$, the coupling depends only on the longitudinal component of the momentum because the energy of LLL for quarks depends only on the longitudinal component of the momentum. Therefore the dependence of the magnetic field on the running coupling is given by

$$
\alpha_{s}^{\|}(e B)=\frac{g^{2}}{4 \pi}=\frac{1}{\alpha_{s}^{0}\left(\mu_{0}\right)^{-1}+\frac{11 N_{c}}{12 \pi} \ln \left(\frac{\Lambda_{Q C D}^{2}+M_{B}^{2}}{\mu_{0}^{2}}\right)+\frac{1}{3 \pi} \sum_{f} \frac{\left|q_{f} B\right|}{\tau}},
$$

where

$$
\alpha_{s}^{0}\left(\mu_{0}\right)=\frac{12 \pi}{11 N_{c} \ln \left(\frac{\mu_{0}^{2}+M_{B}^{2}}{\Lambda_{V}^{2}}\right)}
$$

and $M_{B}$ is taken as an infrared mass that can be interpreted as the ground-state mass of two gluons connected by the fundamental string $(\sim 1 \mathrm{GeV})$, with the string tension, $\tau=0.18 \mathrm{GeV}^{2}, \Lambda_{V}$ and $\mu_{0}$ are taken as $0.385 \mathrm{GeV}$ and $1.1 \mathrm{GeV}$, respectively [56-58].

We now revisit the quark and gluon propagators at the vacuum in a strong magnetic field and then thermalize them in a hot QCD medium, which are the ingredients to compute the quark self-energy.

\subsection{Vacuum propagators in a strong magnetic field}

The presence of the magnetic field breaks the translational invariance of the space, as a result the quark propagator factorizes into the components of momentum, which are transverse and longitudinal to the magnetic field. Schwinger's proper-time method [54] computes the quark propagator in coordinate-space as

$$
S(x, y)=\phi(x, y) \int \frac{d^{4} K}{(2 \pi)^{4}} S(K) e^{-i K(x-y)},
$$

where the phase factor, $\phi(x, y)$ is expressed as

$$
\phi(x, y)=e^{i\left|q_{f}\right| \int_{y}^{x} A^{\mu}(\zeta) d \zeta_{\mu}} .
$$


The phase factor is the gauge-dependent part, which is responsible for the breaking of the translational invariance. However, for a single-fermion line, it is possible to gauge away the phase factor for a symmetric gauge $\left(A^{\mu}(x)=\frac{B}{2}\left(0,-x_{2}, x_{1}, 0\right)\right)$ by an appropriate gauge transformation, so one can work with the momentum-space representation [59] as an integral over the proper-time $(s)$,

$$
\begin{aligned}
S(K)= & i \int_{0}^{\infty} d s e^{-i s m_{f}^{2}} \exp \left(i s k_{\|}^{2}-\frac{i k_{\perp}^{2} \tan \left(\left|q_{f} B s\right|\right)}{\left|q_{f} B\right|}\right) \\
& \times\left[\left(m_{f}+\gamma^{\|} \cdot k_{\|}\right)\left(1+\gamma^{1} \gamma^{2} \tan \left(\left|q_{f} B s\right|\right)\right)-\gamma^{\perp} \cdot k_{\perp}\left(1+\tan ^{2}\left(\left|q_{f} B s\right|\right)\right)\right],
\end{aligned}
$$

where the variables in the above equation are defined as follows,

$$
\begin{aligned}
k_{\|} & \equiv\left(k_{0}, 0,0, k_{3}\right), & k_{\perp} & \equiv\left(0, k_{1}, k_{2}, 0\right), \\
\gamma^{\|} & \equiv\left(\gamma^{0}, \gamma^{3}\right), & \gamma^{\perp} & \equiv\left(\gamma^{1}, \gamma^{2}\right), \\
g^{\mu \nu} & =g_{\|}^{\mu \nu}+g_{\perp}^{\mu \nu}, & & \\
g_{\|}^{\mu \nu} & =\operatorname{diag}(1,0,0,-1), & g_{\perp}^{\mu \nu} & =\operatorname{diag}(0,-1,-1,0), \\
\gamma^{\|} \cdot k_{\|} & =\gamma^{0} k_{0}-\gamma^{3} k_{3}, & \gamma^{\perp} \cdot k_{\perp} & =\gamma^{1} k_{1}+\gamma^{2} k_{2}, \\
k_{\|}^{2} & \equiv k_{0}^{2}-k_{3}^{2}, & k_{\perp}^{2} & \equiv k_{1}^{2}+k_{2}^{2} .
\end{aligned}
$$

Finally after integrating over the proper-time, the propagator in the momentum-space, $S(K)$ can be obtained in the discrete form,

$$
S(K)=i e^{-\frac{k_{\perp}^{2}}{\left|q_{f} B\right|}} \sum_{n=0}^{\infty}(-1)^{n} \frac{D_{n}\left(\left|q_{f} B\right|, K\right)}{k_{\|}^{2}-m_{f}^{2}-2\left|q_{f} B\right| n},
$$

where $D_{n}\left(\left|q_{f} B\right|, K\right)$ 's are the generalized Laguerre polynomials, labeling the Landau levels $[55,60,61]$ as

$$
\begin{aligned}
D_{n}\left(\left|q_{f} B\right|, K\right)= & \left(\gamma^{\|} \cdot k_{\|}+m_{f}\right)\left[\left(1-i \gamma^{1} \gamma^{2}\right) L_{n}\left(\frac{2 k_{\perp}^{2}}{\left|q_{f} B\right|}\right)-\left(1+i \gamma^{1} \gamma^{2}\right) L_{n-1}\left(\frac{2 k_{\perp}^{2}}{\left|q_{f} B\right|}\right)\right] \\
& +4 \gamma^{\perp} \cdot k_{\perp} L_{n-1}^{(1)}\left(\frac{2 k_{\perp}^{2}}{\left|q_{f} B\right|}\right) .
\end{aligned}
$$

In the presence of a strong magnetic field $\left(k_{\|}^{2}, k_{\perp}^{2} \ll\left|q_{f} B\right|\right)$, the transitions to the higher Landau levels $(n \geq 1)$ are suppressed, so only LLL $(n=0)$ is occupied. Therefore the quark propagator in the SMF limit reduces to

$$
S_{L L L}(K)=i e^{-\frac{k_{\perp}^{2}}{\left|q_{f} B\right|}} \frac{\left(\gamma^{\|} \cdot k_{\|}+m_{f}\right)}{k_{\|}^{2}-m_{f}^{2}+i \epsilon}\left(1-\gamma^{0} \gamma^{3} \gamma^{5}\right) .
$$

However, the gluons being uncharged particles remain unaffected by the magnetic field, hence the form of the vacuum gluon propagator in the Feynman gauge remains the same even in the presence of a magnetic field as

$$
D_{0}^{\mu \nu}(Q)=\frac{i g^{\mu \nu}}{Q^{2}+i \epsilon}
$$




\subsection{Thermalization of propagators in a strong magnetic field}

The vacuum quark and gluon propagators in the presence of a strong magnetic field discussed above get thermalized in a thermal QCD medium using the real time formalism, where the medium effects are introduced via the distribution functions. In this formalism, the propagators are manifestly separated into the vacuum and thermal parts at the price of doubling the degrees of freedom, as a result the propagators acquire $(2 \times 2)$ matrix structures. In an equilibrated medium, it is enough to evaluate the one-loop quark self-energy with the 11-components of the quark and gluon propagators.

\subsubsection{Quark propagator}

As we know that in SMF limit the quarks are only populated in the LLL limit, so we are interested in thermalizing the quark propagator in LLL only. Denoting $S_{L L L}(K)$ by $S_{0}(K)$, the vacuum quark propagator gets a matrix structure in the thermal medium as

$$
S(K)=U_{F}\left(k_{0}\right)\left(\begin{array}{cc}
S_{0}(K) & 0 \\
0 & S_{0}^{*}(K)
\end{array}\right) U_{F}\left(k_{0}\right),
$$

where the unitary matrix, $U_{F}\left(k_{0}\right)$ brings the temperature-dependence into the vacuum propagator through the distribution function as

$$
U_{F}\left(k_{0}\right)=\left(\begin{array}{cc}
\sqrt{1-n_{F}\left(k_{0}\right)} & -\sqrt{n_{F}\left(k_{0}\right)} \\
\sqrt{n_{F}\left(k_{0}\right)} & \sqrt{1-n_{F}\left(k_{0}\right)}
\end{array}\right),
$$

where the quark distribution function is given by

$$
n_{F}\left(k_{0}\right)=\frac{1}{e^{\beta\left|k_{0}\right|}+1} .
$$

Thus substituting the unitary matrix into eq. (2.11), we simplify the thermalized matrix propagator for the quarks,

$$
S(K)=\left(\begin{array}{cc}
n_{2}^{2} S_{0}(K)-n_{1}^{2} S_{0}^{*}(K) & -n_{1} n_{2}\left(S_{0}(K)+S_{0}^{*}(K)\right) \\
n_{1} n_{2}\left(S_{0}(K)+S_{0}^{*}(K)\right) & n_{2}^{2} S_{0}^{*}(K)-n_{1}^{2} S_{0}(K)
\end{array}\right),
$$

where $n_{1}$ and $n_{2}$ are denoted by $n_{1}=\sqrt{n_{F}\left(k_{0}\right)}$ and $n_{2}=\sqrt{1-n_{F}\left(k_{0}\right)}$, respectively.

Thus the 11-component of the propagator matrix for quarks in the thermal medium can be read off,

$$
\begin{aligned}
S_{11}(K)= & i e^{-\frac{k_{\perp}^{2}}{\left|q_{f} B\right|}\left(\gamma^{0} k_{0}-\gamma^{3} k_{3}+m_{f}\right)\left(1-\gamma^{0} \gamma^{3} \gamma^{5}\right)} \\
& \times\left[\frac{1}{k_{\|}^{2}-m_{f}^{2}+i \epsilon}+2 \pi i n_{F}\left(k_{0}\right) \delta\left(k_{\|}^{2}-m_{f}^{2}\right)\right],
\end{aligned}
$$

which is found to be modified by the magnetic field. 


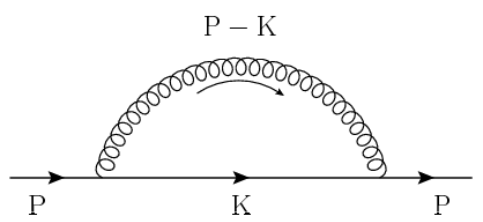

Figure 1. Quark self-energy.

\subsubsection{Gluon propagator}

Similarly the unitary matrix needed to thermalize the gluon propagator in the vacuum is given by distribution function,

$$
U_{B}\left(q_{0}\right)=\left(\begin{array}{cc}
\sqrt{1+n_{B}\left(q_{0}\right)} & \sqrt{n_{B}\left(q_{0}\right)} \\
\sqrt{n_{B}\left(q_{0}\right)} & \sqrt{1+n_{B}\left(q_{0}\right)}
\end{array}\right),
$$

where the distribution function for gluons is given by

$$
n_{B}\left(q_{0}\right)=\frac{1}{e^{\beta\left|q_{0}\right|}-1} .
$$

Hence the propagator matrix for gluons in the thermal medium acquires the following form,

$$
D^{\mu \nu}(Q)=U_{B}\left(q_{0}\right)\left(\begin{array}{cc}
D_{0}^{\mu \nu}(Q) & 0 \\
0 & D_{0}^{* \mu \nu}(Q)
\end{array}\right) U_{B}\left(q_{0}\right),
$$

where $D_{0}^{\mu \nu}(Q)$ is the vacuum gluon propagator (2.10). Hence the 11-component of the propagator matrix for gluons is given by

$$
D_{11}^{\mu \nu}(Q)=i g^{\mu \nu}\left[\frac{1}{Q^{2}+i \epsilon}-2 \pi i n_{B}\left(q_{0}\right) \delta\left(Q^{2}\right)\right] .
$$

\subsection{One-loop quark self-energy in a strongly magnetized medium}

Using the 11-components of the quark and gluon matrix propagators from equations (2.15) and (2.19), respectively, we can obtain the 11-component of the quark self-energy matrix from the one-loop diagram (figure 1) in a strong magnetic field,

$$
\Sigma(P)=-\frac{4}{3} g^{2} i \int \frac{d^{4} K}{(2 \pi)^{4}}\left[\gamma_{\mu} S_{11}(K) \gamma^{\mu} D_{11}(P-K)\right],
$$

where $4 / 3$ is the QCD colour factor and $g$ is the QCD running coupling.

In the presence of the magnetic field, the momentum integration is factorized into the parallel and perpendicular components with respect to the direction of magnetic field, so the above self-energy is rewritten as

$$
\begin{aligned}
\Sigma(P)= & \frac{4 g^{2} i}{3(2 \pi)^{4}} \int d^{2} k_{\perp} d^{2} k_{\|} e^{-\frac{k_{\perp}^{2}}{\operatorname{qu}_{f} B \mid}}\left[\gamma_{\mu}\left(\gamma^{0} k_{0}-\gamma^{3} k_{3}+m_{f}\right)\left(1-\gamma^{0} \gamma^{3} \gamma^{5}\right) \gamma^{\mu}\right] \\
& \times\left[\frac{1}{k_{\|}^{2}-m_{f}^{2}+i \epsilon}+2 \pi i n_{F}\left(k_{0}\right) \delta\left(k_{\|}^{2}-m_{f}^{2}\right)\right] \\
& \times\left[\frac{1}{(P-K)^{2}+i \epsilon}-2 \pi i n_{B}\left(p_{0}-k_{0}\right) \delta\left((P-K)^{2}\right)\right]
\end{aligned}
$$


where the trace of gamma matrices is calculated as

$$
\gamma_{\mu}\left(\gamma^{0} k_{0}-\gamma^{3} k_{3}+m_{f}\right)\left(1-\gamma^{0} \gamma^{3} \gamma^{5}\right) \gamma^{\mu}=-2\left[J_{+}\left(\gamma^{0} k_{0}-\gamma^{3} k_{3}\right)-2 m_{f}\right]
$$

where, $J_{+}$is given by

$$
J_{+}=1+\gamma^{0} \gamma^{3} \gamma^{5}
$$

In SMF limit, both $k_{\|}^{2}$ and $k_{\perp}^{2}$ are assumed to be much smaller than $\left|q_{f} B\right|$, hence, the form factor - $\exp \left(-k_{\perp}^{2} /\left|q_{f} B\right|\right)$ can be approximately equal to 1 and the upper limits of the momentum integrals may be cut off at $\left|q_{f} B\right|$. Moreover, the external quark momentum $P$ can be assumed to be purely longitudinal [62] in LLL approximation, i.e. $p_{\perp} \approx 0$, so the square of internal gluon-momentum is approximated as

$$
(P-K)^{2} \simeq\left(p_{\|}-k_{\|}\right)^{2}-k_{\perp}^{2}
$$

Thus the quark self-energy (2.21) becomes the function of the longitudinal component of momentum only and is separated into the vacuum and the medium components,

$$
\begin{aligned}
\Sigma\left(p_{\|}\right)= & \frac{-8 g^{2} i}{3(2 \pi)^{4}} \int d^{2} k_{\perp} d^{2} k_{\|}\left[J_{+}\left(\gamma^{0} k_{0}-\gamma^{3} k_{3}\right)-2 m_{f}\right] \\
& \times\left[\frac{1}{k_{\|}^{2}-m_{f}^{2}+i \epsilon}+2 \pi i n_{F}\left(k_{0}\right) \delta\left(k_{\|}^{2}-m_{f}^{2}\right)\right] \\
& \times\left[\frac{1}{\left(p_{\|}-k_{\|}\right)^{2}-k_{\perp}^{2}+i \epsilon}-2 \pi i n_{B}\left(p_{0}-k_{0}\right) \delta\left(\left(p_{\|}-k_{\|}\right)^{2}-k_{\perp}^{2}\right)\right] \\
\equiv & \Sigma_{V}\left(p_{\|}\right)+\Sigma_{n}\left(p_{\|}\right)+\Sigma_{n^{2}}\left(p_{\|}\right),
\end{aligned}
$$

where $\Sigma_{V}\left(p_{\|}\right)$denotes the vacuum contribution and both $\Sigma_{n}\left(p_{\|}\right)$as well as $\Sigma_{n^{2}}\left(p_{\|}\right)$represent the medium contributions, depending on the distribution function linearly and quadratically, respectively.

\subsubsection{Vacuum contribution}

We will now calculate the vacuum contribution to the quark self-energy from eq. (2.25).

$$
\begin{aligned}
\Sigma_{V}\left(p_{\|}\right)= & \frac{-8 g^{2} i}{3(2 \pi)^{4}} \int d^{2} k_{\perp} d^{2} k_{\|}\left[J_{+}\left(\gamma^{0} k_{0}-\gamma^{3} k_{3}\right)-2 m_{f}\right] \\
& \times\left[\frac{1}{k_{\|}^{2}-m_{f}^{2}+i \epsilon}\right]\left[\frac{1}{\left(p_{\|}-k_{\|}\right)^{2}-k_{\perp}^{2}+i \epsilon}\right] .
\end{aligned}
$$

Separating the real and imaginary parts by the identity,

$$
\frac{1}{x \pm y \pm i \epsilon}=\mathrm{P}\left(\frac{1}{x \pm y}\right) \mp i \pi \delta(x \pm y)
$$


we obtain the real part,

$$
\begin{aligned}
\Sigma_{V}\left(p_{\|}\right) & =\frac{-8 g^{2}}{3(2 \pi)^{4}} \int d^{2} k_{\perp} \int d^{2} k_{\|}\left[J_{+} \gamma^{\|} \cdot p_{\|}-2 m_{f}\right] \frac{i}{\left[k_{\|}^{2}-m_{f}^{2}\right]\left[\left(p_{\|}-k_{\|}\right)^{2}-k_{\perp}^{2}\right]} \\
& \equiv \frac{-8 g^{2}}{3(2 \pi)^{4}} \int d^{2} k_{\perp} I .
\end{aligned}
$$

Using the Feynman parametrization,

$$
\frac{i}{\left[\left(p_{\|}-k_{\|}\right)^{2}-k_{\perp}^{2}\right]\left[k_{\|}^{2}-m_{f}^{2}\right]}=\int_{0}^{1} d z \frac{i}{\left[\left\{\left(p_{\|}-k_{\|}\right)^{2}-k_{\perp}^{2}\right\} z+\left(k_{\|}^{2}-m_{f}^{2}\right)(1-z)\right]^{2}}
$$

and changing the variable from $k_{\|}$to $k^{\prime}$ by $k_{\|}-z p_{\|}=k^{\prime}$, the integral, $I$ can be rewritten by the Wick rotation,

$$
\begin{aligned}
I & =-\int_{0}^{1} d z d^{2} k^{\prime} \frac{z J_{+} \gamma^{\|} \cdot p_{\|}-2 m_{f}}{\left[k^{\prime 2}-z(1-z) p_{\|}^{2}+z k_{\perp}^{2}+(1-z) m_{f}^{2}\right]^{2}} \\
& =\frac{\pi}{\left|q_{f} B\right|} \int_{0}^{1} d z\left(z J_{+} \gamma^{\|} \cdot p_{\|}-2 m_{f}\right)+\pi \int_{0}^{1} d z \frac{-z J_{+} \gamma^{\|} \cdot p_{\|}+2 m_{f}}{-z(1-z) p_{\|}^{2}+z k_{\perp}^{2}+(1-z) m_{f}^{2}} \\
& \equiv I_{1}+I_{2}
\end{aligned}
$$

where the first integral $\left(I_{1}\right)$ is solved analytically as

$$
\begin{aligned}
I_{1} & =\frac{\pi}{\left|q_{f} B\right|} \int_{0}^{1} d z\left(z J_{+} \gamma^{\|} \cdot p_{\|}-2 m_{f}\right) \\
& =\frac{\pi}{2\left|q_{f} B\right|}\left(J_{+} \gamma^{\|} \cdot p_{\|}-4 m_{f}\right) .
\end{aligned}
$$

For the second integral $\left(I_{2}\right)$, we expand it in a Taylor series around the mass-shell condition $-\gamma^{\|} \cdot p_{\|}=m_{f}$ in a strong magnetic field,

$$
I_{2}=A+B\left(\gamma^{\|} \cdot p_{\|}-m_{f}\right)+C\left(\gamma^{\|} \cdot p_{\|}-m_{f}\right)^{2}+\cdots .
$$

Dropping the higher-order terms, $I_{2}$ can be written as

$$
I_{2}=A+B\left(\gamma^{\|} \cdot p_{\|}-m_{f}\right),
$$

where the coefficients, $A$ and $B$ are determined from the following conditions,

$$
\begin{aligned}
A & =\left.I_{2}\right|_{\gamma \| \cdot p_{\|}=m_{f}} \\
& =-\pi m_{f} \int_{0}^{1} d z \frac{z J_{+}-2}{m_{f}^{2}(1-z)^{2}+z k_{\perp}^{2}}, \\
B & =\left.\frac{\partial I_{2}}{\partial\left(\gamma^{\|} \cdot p_{\|}\right)}\right|_{\gamma \| \cdot p_{\|}=m_{f}} \\
& =-\pi \int_{0}^{1} d z \frac{z J_{+}}{(1-z)^{2} m_{f}^{2}+z k_{\perp}^{2}}-\pi \int_{0}^{1} d z \frac{2 m_{f}^{2} z(1-z)\left(z J_{+}-2\right)}{\left\{(1-z)^{2} m_{f}^{2}+z k_{\perp}^{2}\right\}^{2}}
\end{aligned}
$$


After the Feynman integrations in equations (2.34) and (2.35), the coefficients $A$ and $B$ are found to obtain the terms in powers of the quark masses $\left(m_{f}\right)$, so, at least, for two light flavors, we may drop the terms proportional to $m_{f}^{2}$ and its higher orders to simplify their forms as

$$
\begin{aligned}
A & \simeq-\frac{\pi J_{+}}{2 m_{f}} \ln \left(\frac{k_{\perp}^{2}}{m_{f}^{2}}\right), \\
B & \simeq \frac{\pi J_{+}}{2 m_{f}^{2}} \ln \left(\frac{k_{\perp}^{2}}{m_{f}^{2}}\right) .
\end{aligned}
$$

Thus we obtain the second integral, $I_{2}$ in a simplified form,

$$
I_{2}=-\frac{\pi J_{+}}{m_{f}} \ln \left(\frac{k_{\perp}^{2}}{m_{f}^{2}}\right)+\frac{\pi J_{+}}{2 m_{f}^{2}}\left(\gamma^{\|} \cdot p_{\|}\right) \ln \left(\frac{k_{\perp}^{2}}{m_{f}^{2}}\right) .
$$

Finally the $k_{\|}$-integration in the self-energy (2.30) yields as

$$
I=\frac{\pi}{2\left|q_{f} B\right|}\left(J_{+} \gamma^{\|} \cdot p_{\|}-4 m_{f}\right)-\frac{\pi J_{+}}{m_{f}} \ln \left(\frac{k_{\perp}^{2}}{m_{f}^{2}}\right)+\frac{\pi J_{+}}{2 m_{f}^{2}}\left(\gamma^{\|} \cdot p_{\|}\right) \ln \left(\frac{k_{\perp}^{2}}{m_{f}^{2}}\right)
$$

which, in turn gives the real part of the vacuum contribution from eq. (2.28),

$$
\begin{aligned}
\Sigma_{V}\left(p_{\|}\right)= & \frac{-8 g^{2} \pi}{3(2 \pi)^{4}} \int_{0}^{\left|q_{f} B\right|} d k_{\perp}^{2} I \\
= & \frac{J_{+}\left(\gamma^{\|} \cdot p_{\|}\right) g^{2}}{6 \pi^{2}}\left[-\frac{1}{2}-\frac{\left|q_{f} B\right|}{2 m_{f}^{2}}\left\{\ln \left(\frac{\left|q_{f} B\right|}{m_{f}^{2}}\right)-1\right\}\right] \\
& +\frac{g^{2}}{6 \pi^{2}}\left[2 m_{f}+\frac{J_{+}\left|q_{f} B\right|}{m_{f}}\left\{\ln \left(\frac{\left|q_{f} B\right|}{m_{f}^{2}}\right)-1\right\}\right] .
\end{aligned}
$$

\subsubsection{Medium contribution}

We will first calculate the medium contribution, $\Sigma_{n}\left(p_{\|}\right)$, which depends on the quark and gluon distribution functions linearly,

$$
\begin{aligned}
\Sigma_{n}\left(p_{\|}\right)= & \frac{8 g^{2}}{3(2 \pi)^{3}} \int d^{2} k_{\perp} d k_{3} d k_{0}\left[J_{+}\left(\gamma^{0} k_{0}-\gamma^{3} k_{3}\right)-2 m_{f}\right] \\
& \times\left[\frac{\delta\left(k_{\|}^{2}-m_{f}^{2}\right) n_{F}\left(k_{0}\right)}{\left(p_{\|}-k_{\|}\right)^{2}-k_{\perp}^{2}}+\frac{\delta\left(\left(p_{\|}-k_{\|}\right)^{2}-k_{\perp}^{2}\right)\left[-n_{B}\left(p_{0}-k_{0}\right)\right]}{k_{\|}^{2}-m_{f}^{2}}\right] \\
\equiv & \Sigma_{n_{F}}\left(p_{\|}\right)+\Sigma_{n_{B}}\left(p_{\|}\right) .
\end{aligned}
$$

The contribution due to the quark distribution function is rewritten by

$$
\Sigma_{n_{F}}\left(p_{\|}\right)=\frac{8 g^{2}}{3(2 \pi)^{3}} \int d^{2} k_{\perp} d k_{3} d k_{0}\left[J_{+}\left(\gamma^{0} k_{0}-\gamma^{3} k_{3}\right)-2 m_{f}\right] \frac{\delta\left(k_{0}^{2}-\omega_{k}^{2}\right) n_{F}\left(k_{0}\right)}{\left(p_{\|}-k_{\|}\right)^{2}-k_{\perp}^{2}}
$$


with $\omega_{k}^{2}=k_{3}^{2}+m_{f}^{2}$. After integrating over $k_{0}$, we are left with the remaining integrations over $k_{3}$ and $k_{\perp}$ momenta,

$$
\Sigma_{n_{F}}\left(p_{\|}\right)=\frac{-8 g^{2}}{3(2 \pi)^{3}} \int d k_{3} \frac{n_{F}\left(\omega_{k}\right)}{\omega_{k}}\left(J_{+} \gamma^{3} k_{3}+2 m_{f}\right) \int d^{2} k_{\perp} \frac{1}{\left(p_{\|}-m_{f}\right)^{2}-k_{\perp}^{2}},
$$

where the $k_{3}$-integration is solved [63] in power of $m_{f} / T$,

$$
I_{k_{3}}=4 m_{f}\left[-\frac{1}{2} \ln \left(\frac{m_{f}}{\pi T}\right)-\frac{1}{2} \gamma_{E}+\mathcal{O}\left(\frac{m_{f}^{2}}{T^{2}}\right)\right],
$$

where $\gamma_{E}$ is the Euler-Mascheroni constant.

For the temperatures achieved in the current heavy ion collisions, the masses of the two light flavors $\left(u\right.$ and $d$ ) are much smaller than the temperature of the medium (i.e. $m_{f} \ll T$ ), so the higher-order terms, $\mathcal{O}\left(m_{f}^{2} / T^{2}\right)$ may be dropped to get a simple form for $I_{k_{3}}$,

$$
I_{k_{3}}=-2 m_{f}\left[\ln \left(\frac{m_{f}}{\pi T}\right)+\gamma_{E}\right] .
$$

Now the remaining $k_{\perp}$-integration in eq. (2.43) is performed by, using the LLL approximation,

$$
\begin{aligned}
I_{k_{\perp}} & =\pi \int_{0}^{\left|q_{f} B\right|} d k_{\perp}^{2} \frac{1}{\left(p_{\|}-m_{f}\right)^{2}-k_{\perp}^{2}} \\
& =-\pi\left[i \pi+\ln \left(\frac{\left|q_{f} B\right|}{\left(p_{\|}-m_{f}\right)^{2}}\right)\right] .
\end{aligned}
$$

Thus, substituting the values of $I_{k_{3}}$ and $I_{k_{\perp}}$, the contribution due to the quark distribution function (2.43) is given by

$$
\Sigma_{n_{F}}\left(p_{\|}\right)=-\frac{2 g^{2} m_{f}}{3 \pi^{2}} \ln \left(\frac{\left|q_{f} B\right|}{\left(p_{\|}-m_{f}\right)^{2}}\right)\left[\ln \left(\frac{m_{f}}{\pi T}\right)+\gamma_{E}\right] .
$$

Similarly the medium contribution involving the gluon distribution function, $\Sigma_{n_{B}}\left(p_{\|}\right)$ in eq. (2.41) is rewritten as

$$
\begin{aligned}
\Sigma_{n_{B}}\left(p_{\|}\right)=-\frac{8 g^{2}}{3(2 \pi)^{3}} & \int d^{2} k_{\perp} d k_{3} d k_{0}\left[J_{+}\left(\gamma^{0} k_{0}-\gamma^{3} k_{3}\right)-2 m_{f}\right] \\
& \times \frac{\delta\left(\left(p_{\|}-k_{\|}\right)^{2}-k_{\perp}^{2}\right)}{k_{0}^{2}-\omega_{k}^{2}} n_{B}\left(p_{0}-k_{0}\right) .
\end{aligned}
$$

Using the roots of the equation,

$$
\left(p_{\|}-k_{\|}\right)^{2}-k_{\perp}^{2}=0
$$

the above Dirac delta function is approximated by, for small values of $k_{\perp}$,

$$
\delta\left(\left(p_{\|}-k_{\|}\right)^{2}-k_{\perp}^{2}\right) \approx \frac{\left[\delta\left\{k_{0}-\left(p_{0}+p_{3}-k_{3}\right)\right\}+\delta\left\{k_{0}-\left(p_{0}-p_{3}+k_{3}\right)\right\}\right]}{2\left(p_{3}-k_{3}\right)} .
$$


Thus, using the above Dirac-delta function, we have performed the $k_{0}$ and $k_{\perp}$ integrations and got the result for the self-energy (2.48), after the final $k_{3}$ integration,

$$
\begin{aligned}
\Sigma_{n_{B}}\left(p_{\|}\right)= & -\frac{4 \pi\left|q_{f} B\right| g^{2}}{3(2 \pi)^{3}} \int_{-\infty}^{+\infty} d k_{3} \frac{n_{B}\left(p_{3}-k_{3}\right)}{\left(p_{3}-k_{3}\right)}\left[\frac{J_{+} \gamma^{0} p_{0}-2 m_{f}}{\left(p_{0}+p_{3}-k_{3}\right)^{2}-\omega_{k}^{2}}\right. \\
& +\frac{J_{+} \gamma^{0}\left(p_{3}-k_{3}\right)}{\left(p_{0}+p_{3}-k_{3}\right)^{2}-\omega_{k}^{2}}-\frac{J_{+} \gamma^{3} k_{3}}{\left(p_{0}+p_{3}-k_{3}\right)^{2}-\omega_{k}^{2}}+\frac{J_{+} \gamma^{0} p_{0}-2 m_{f}}{\left(p_{0}-p_{3}+k_{3}\right)^{2}-\omega_{k}^{2}} \\
& \left.-\frac{J_{+} \gamma^{0}\left(p_{3}-k_{3}\right)}{\left(p_{0}-p_{3}+k_{3}\right)^{2}-\omega_{k}^{2}}-\frac{J_{+} \gamma^{3} k_{3}}{\left(p_{0}-p_{3}+k_{3}\right)^{2}-\omega_{k}^{2}}\right] \\
= & -\frac{4 \pi\left|q_{f} B\right| g^{2}}{3(2 \pi)^{3}} \sum_{i=1}^{6} I^{i},
\end{aligned}
$$

where $I^{i}$ s are found as follows,

$$
\begin{aligned}
I^{1} & =\frac{i \pi\left(J_{+} \gamma^{0} p_{0}-2 m_{f}\right)}{2\left(p_{0}+p_{3}\right)}\left[\frac{\beta\left(a-p_{3}\right)-2}{2 \beta\left(p_{3}-a\right)^{2}}+\frac{n_{B}\left(a-p_{3}\right)}{a-p_{3}}\right], \\
I^{2} & =\frac{-i \pi J_{+} \gamma^{0}}{2\left(p_{0}+p_{3}\right)}\left[\frac{1}{\beta\left(p_{3}-a\right)}+n_{B}\left(a-p_{3}\right)\right] \\
I^{3} & =\frac{-i \pi J_{+} \gamma^{3}}{2\left(p_{0}+p_{3}\right)}\left[\frac{-2 a-\beta p_{3}\left(p_{3}-a\right)}{2 \beta\left(p_{3}-a\right)^{2}}+\frac{a n_{B}\left(a-p_{3}\right)}{a-p_{3}}\right], \\
I^{4} & =\frac{-i \pi\left(J_{+} \gamma^{0} p_{0}-2 m_{f}\right)}{2\left(p_{0}-p_{3}\right)}\left[\frac{\beta\left(b-p_{3}\right)-2}{2 \beta\left(p_{3}-b\right)^{2}}+\frac{n_{B}\left(b-p_{3}\right)}{b-p_{3}}\right], \\
I^{5} & =\frac{-i \pi J_{+} \gamma^{0}}{2\left(p_{0}-p_{3}\right)}\left[\frac{1}{\beta\left(p_{3}-b\right)}+n_{B}\left(b-p_{3}\right)\right] \\
I^{6} & =\frac{i \pi J_{+} \gamma^{3}}{2\left(p_{0}-p_{3}\right)}\left[\frac{-2 b-\beta p_{3}\left(p_{3}-b\right)}{2 \beta\left(p_{3}-b\right)^{2}}+\frac{b n_{B}\left(b-p_{3}\right)}{b-p_{3}}\right]
\end{aligned}
$$

where $a$ and $b$ are given by

$$
\begin{aligned}
& a=\frac{\left(p_{0}+p_{3}\right)^{2}-m_{f}^{2}}{2\left(p_{0}+p_{3}\right)}, \\
& b=\frac{\left(p_{0}-p_{3}\right)^{2}-m_{f}^{2}}{2\left(p_{3}-p_{0}\right)} .
\end{aligned}
$$

Therefore, the medium contribution due to the gluon distribution, $\Sigma_{n_{B}}\left(p_{\|}\right)$does not contribute to the real part of the one-loop quark self-energy.

The medium contribution, involving the product of quark and gluon distribution functions (from eq. (2.25)),

$$
\begin{aligned}
\Sigma_{n^{2}}\left(p_{\|}\right)= & \frac{-8 g^{2} i}{3(2 \pi)^{4}} \int d^{2} k_{\perp} d^{2} k_{\|} 4 \pi^{2} n_{F}\left(k_{0}\right) n_{B}\left(p_{0}-k_{0}\right)\left[J_{+}\left(\gamma^{0} k_{0}-\gamma^{3} k_{3}\right)-2 m_{f}\right] \\
& \times\left[\delta\left(k_{\|}^{2}-m_{f}^{2}\right) \delta\left(\left(p_{\|}-k_{\|}\right)^{2}-k_{\perp}^{2}\right)\right]
\end{aligned}
$$

is purely imaginary, so it does not contribute to the real part of the quark self-energy. 
Thus the vacuum (2.40) and the medium (2.47) contributions are added together to give the real part of the one-loop quark self-energy in a strong magnetic field,

$$
\begin{aligned}
\Sigma\left(p_{\|}\right)= & J_{+}\left(\gamma^{\|} \cdot p_{\|}\right) \frac{g^{2}}{6 \pi^{2}}\left[-\frac{1}{2}-\frac{\left|q_{f} B\right|}{2 m_{f}^{2}}\left\{\ln \left(\frac{\left|q_{f} B\right|}{m_{f}^{2}}\right)-1\right\}\right] \\
& +\frac{g^{2}}{6 \pi^{2}}\left[2 m_{f}+\frac{J_{+}\left|q_{f} B\right|}{m_{f}}\left\{\ln \left(\frac{\left|q_{f} B\right|}{m_{f}^{2}}\right)-1\right\}\right] \\
& -\frac{2 g^{2} m_{f}}{3 \pi^{2}} \ln \left(\frac{\left|q_{f} B\right|}{\left(p_{\|}-m_{f}\right)^{2}}\right)\left[\ln \left(\frac{m_{f}}{\pi T}\right)+\gamma_{E}\right],
\end{aligned}
$$

which enables us to compute the effective quark propagator from the Schwinger-Dyson equation (2.1).

\section{The effective gluon propagator in a strongly-magnetized hot QCD medium}

This section is devoted to calculate the effective gluon propagator in a thermal medium in the presence of a strong magnetic field, which can be obtained from the Schwinger-Dyson equation,

$$
D_{\mu \nu}^{-1}(P)=D_{0 \mu \nu}^{-1}(P)+\Pi_{\mu \nu}(P) .
$$

At finite temperature, the effective gluon propagator is decomposed into the longitudinal and transverse components due to the splitting of the gluon self-energy tensor into the longitudinal and transverse components in the rest frame of the heat bath. Although the gluons are not affected directly by the magnetic field but the implicit dependence of magnetic field enters directly through the Debye mass and indirectly through the running strong coupling. Therefore, we first revisit the gluon self-energy in a thermal medium in the coming subsection.

\subsection{One-loop gluon self-energy in a hot QCD medium}

In the vacuum, the gluon self-energy tensor may be constructed by the linear combination $\left(P_{\mu} P_{\nu}\right)$ of four-momentum $\left(P_{\mu}\right)$ and the metric tensor $\left(g_{\mu \nu}\right)$. Therefore, it only depends on one scalar function, which is a function of the Lorentz invariant quantity, $P^{2}$. Further imposition of the Ward identity gives the structure of the tensor,

$$
\begin{aligned}
\Pi_{\mu \nu}(P) & =\left(g_{\mu \nu}-\frac{P_{\mu} P_{\nu}}{P^{2}}\right) \Pi\left(P^{2}\right) \\
& \equiv P_{\mu \nu} \Pi\left(P^{2}\right)
\end{aligned}
$$

where $P_{\mu \nu}$ is the four-dimensional transverse projection operator.

However, at finite temperature, the Lorentz invariance is broken due to the direction of heat bath, which is introduced by the four-velocity vector, $u_{\mu}$ in the rest frame of the heat bath. Now with the available four-vectors, $P_{\mu}, u_{\mu}$ and the tensor, $g_{\mu \nu}$, the two 
orthogonal tensors, which are compatible to the physical degrees of freedom and project on the subspace transverse (T) and longitudinal (L) to the three-momentum, $\mathbf{p}$, respectively, are constructed as

$$
\begin{aligned}
& P_{\mu \nu}^{T}=g_{\mu \nu}-\frac{P_{\mu} P_{\nu}}{P^{2}}-\frac{P_{\mu \nu}^{L}}{-N^{2}}, \\
& P_{\mu \nu}^{L}=-N_{\mu} N_{\nu}, \quad \text { with } N_{\mu}=\frac{P_{\mu}(P . u)-u_{\mu} P^{2}}{(P . u)^{2}-P^{2}} .
\end{aligned}
$$

The above tensorial projectors facilitate to decompose the gluon self-energy tensor as

$$
\Pi_{\mu \nu}\left(p_{0}, \mathbf{p}\right)=P_{\mu \nu}^{T} \Pi_{T}\left(p_{0}, \mathbf{p}\right)+P_{\mu \nu}^{L} \Pi_{L}\left(p_{0}, \mathbf{p}\right),
$$

where the functions $\Pi_{T}$ and $\Pi_{L}$ are the transverse and longitudinal components of the self-energy, respectively and depend on both the energy and three-momentum in the rest frame of the medium,

$$
\begin{aligned}
p_{0} & =u \cdot P, \\
\mathbf{p} & =\sqrt{(u \cdot P)^{2}-P^{2}} .
\end{aligned}
$$

Utilizing the properties of the projection operators, $P_{\mu \nu}^{L}$, and $P_{\mu \nu}^{T}$, the transverse and longitudinal self-energies can be obtained by the inverse transformation,

$$
\begin{aligned}
& \Pi_{L}(P)=-\Pi_{00}(P), \\
& \Pi_{T}(P)=\frac{1}{2}\left(\Pi_{\mu}^{\mu}(P)-\frac{P^{2}}{\mathbf{p}^{2}} \Pi_{L}(P)\right) .
\end{aligned}
$$

The HTL perturbation theory determines the gluon self-energy tensor by the angular average over the spatial directions of light-like vectors, $K_{\mu}=(1, \hat{\mathbf{k}})$,

$$
\begin{aligned}
\Pi_{\mu \nu}(P) & =m_{D}^{2}\left[\int \frac{d \Omega}{4 \pi} K_{\mu} K_{\nu} \frac{P_{\mu} \cdot u^{\mu}}{P_{\mu} \cdot K^{\mu}}-u_{\mu} u_{\nu}\right] \\
& =m_{D}^{2}\left[\int \frac{d \Omega}{4 \pi} K_{\mu} K_{\nu} \frac{p_{0}}{p_{0}+\mathbf{p} \cdot \hat{\mathbf{k}}}-u_{\mu} u_{\nu}\right],
\end{aligned}
$$

which, in turn gives the transverse and longitudinal components of the gluon self-energy,

$$
\begin{aligned}
& \Pi_{T}(P)=\frac{m_{D}^{2}}{2} \frac{p_{0}^{2}}{\mathbf{p}^{2}}+\frac{m_{D}^{2}}{4} \frac{p_{0}}{\mathbf{p}}\left(1-\frac{p_{0}^{2}}{\mathbf{p}^{2}}\right) \ln \left(\frac{p_{0}+\mathbf{p}}{p_{0}-\mathbf{p}}\right), \\
& \Pi_{L}(P)=m_{D}^{2}-\frac{m_{D}^{2}}{2} \frac{p_{0}}{\mathbf{p}} \ln \left(\frac{p_{0}+\mathbf{p}}{p_{0}-\mathbf{p}}\right),
\end{aligned}
$$

respectively.

Therefore the effective thermal gluon propagator will be obtained by the SchwingerDyson equation,

$$
D_{\mu \nu}(P)=P_{\mu \nu}^{T} \Delta_{T}+P_{\mu \nu}^{L} \frac{P^{2}}{\mathbf{p}^{2}} \Delta_{L}
$$


where the transverse and longitudinal components are given by the respective gluon selfenergies $\left(\Pi_{T}\right.$ and $\left.\Pi_{L}\right)$, respectively,

$$
\begin{aligned}
& \Delta_{T}=\frac{-1}{P^{2}+\Pi_{T}(P)}, \\
& \Delta_{L}=\frac{1}{\mathbf{p}^{2}+\Pi_{L}(P)} .
\end{aligned}
$$

Physically, $\Delta_{T}$ describes the propagation of the two transverse vacuum modes in a thermal medium because $\operatorname{Tr}\left(P^{T}\right)=2$ whereas $\Delta_{L}$ is absent in the vacuum, so it represents the collective modes of the medium.

However, when the medium becomes strongly magnetized, the dependence of the magnetic field in $\Pi_{T}(P)$ and $\Pi_{L}(P)$ originates from the dependence of Debye mass on the magnetic field. Therefore we are now going to derive the Debye mass in a strong magnetic field in the next subsection.

\subsection{Screening mass in a strong magnetic field}

The Debye screening mass manifests in the collective oscillation of the medium through the dispersion relation and is obtained by the static limit of the longitudinal part of the gluon self-energy tensor,

$$
\Pi_{L}\left(p_{0}=0, \mathbf{p} \rightarrow 0\right)=m_{D}^{2}
$$

Out of the four contributing diagrams (tadpole, gluon-loop, ghost-loop and quark-loop) to the gluon self-energy, only the quark-loop diagram (figure 2) is affected by the magnetic field.

Using the Keldysh representation of the real time formalism, the Schwinger's propertime propagator in a strong magnetic field (2.15) for the internal quark line gives the 11-component of the gluon self-energy matrix (omitting the prefix "11"),

$$
\begin{aligned}
\Pi^{\mu \nu}(P)= & -\frac{i g^{2}}{2} \sum_{f} \int \frac{d^{4} K}{(2 \pi)^{4}} \operatorname{tr}\left[\gamma^{\mu} S_{11}(K) \gamma^{\nu} S_{11}(K-P)\right] \\
= & \frac{i g^{2}}{2(2 \pi)^{4}} \sum_{f} \int d^{2} k_{\perp} d^{2} k_{\|} \operatorname{tr}\left[\gamma^{\mu}\left(\gamma^{0} k_{0}-\gamma^{3} k_{3}+m_{f}\right)\left(1-\gamma^{0} \gamma^{3} \gamma^{5}\right)\right. \\
& \left.\times \gamma^{\nu}\left(\gamma^{0} q_{0}-\gamma^{3} q_{3}+m_{f}\right)\left(1-\gamma^{0} \gamma^{3} \gamma^{5}\right)\right] \\
& \times\left[\frac{1}{k_{\|}^{2}-m_{f}^{2}+i \epsilon}+2 \pi i n_{F}\left(k_{0}\right) \delta\left(k_{\|}^{2}-m_{f}^{2}\right)\right] e^{-\frac{k_{\perp}^{2}}{\left|q_{f} B\right|}} \\
& \times\left[\frac{1}{q_{\|}^{2}-m_{f}^{2}+i \epsilon}+2 \pi i n_{F}\left(q_{0}\right) \delta\left(q_{\|}^{2}-m_{f}^{2}\right)\right] e^{-\frac{q_{\perp}^{2}}{\left|q_{f} B\right|}}
\end{aligned}
$$

where the colour factor $(1 / 2)$ arises due to the trace over the colour indices and the internal loop momentum, $(K-P)$ is replaced by $Q$. 


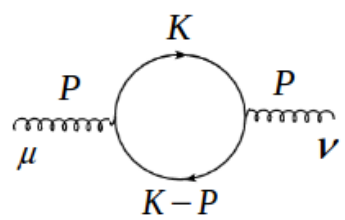

Figure 2. Gluon self-energy.

Due to the presence of the magnetic field, the momentum integration in the self-energy is factorized into the parallel and perpendicular components,

$$
\Pi^{\mu \nu}(P)=\Pi_{k_{\perp}}\left(p_{\perp}\right) \Pi^{\mu \nu}\left(p_{\|}\right) .
$$

So we first integrate the transverse loop-momentum $\left(k_{\perp}\right)$ to obtain the self-energy component as a function of the transverse momentum, $p_{\perp}$ only,

$$
\begin{aligned}
\Pi_{k_{\perp}}\left(p_{\perp}\right) & =\sum_{f} \int d k_{1} d k_{2} e^{-\frac{k_{\perp}^{2}}{\left|q_{f} B\right|}} e^{-\frac{q_{\perp}^{2}}{\left|q_{f} B\right|}} \\
& =\sum_{f} \frac{\pi\left|q_{f} B\right|}{2} e^{-\frac{p_{\perp}^{2}}{2\left|q_{f} B\right|}} .
\end{aligned}
$$

The remaining integration on the longitudinal loop-momentum gives the other component of the self-energy, which is, similar to the quark self-energy, separable into the vacuum and medium contributions,

$$
\begin{aligned}
\Pi^{\mu \nu}\left(p_{\|}\right)= & \frac{i g^{2}}{2(2 \pi)^{4}} \int d k_{0} d k_{3} L^{\mu \nu}\left[\frac{1}{k_{\|}^{2}-m_{f}^{2}+i \epsilon}+2 \pi i n_{F}\left(k_{0}\right) \delta\left(k_{\|}^{2}-m_{f}^{2}\right)\right] \\
& \times\left[\frac{1}{q_{\|}^{2}-m_{f}^{2}+i \epsilon}+2 \pi i n_{F}\left(q_{0}\right) \delta\left(q_{\|}^{2}-m_{f}^{2}\right)\right] \\
\equiv & \Pi_{V}^{\mu \nu}\left(p_{\|}\right)+\Pi_{n}^{\mu \nu}\left(p_{\|}\right)+\Pi_{n^{2}}^{\mu \nu}\left(p_{\|}\right),
\end{aligned}
$$

where the trace over gamma matrices $\left(L^{\mu \nu}\right)$ is calculated as

$$
L^{\mu \nu}=8\left[k_{\|}^{\mu} \cdot q_{\|}^{\nu}+k_{\|}^{\nu} \cdot q_{\|}^{\mu}-g_{\|}^{\mu \nu}\left(k_{\|}^{\mu} \cdot q_{\| \mu}-m_{f}^{2}\right)\right] .
$$

The vacuum contribution is separated from eq. (3.20),

$$
\Pi_{V}^{\mu \nu}\left(p_{\|}\right)=\frac{i g^{2}}{2(2 \pi)^{4}} \int d k_{0} d k_{3} L^{\mu \nu}\left[\frac{1}{\left(k_{\|}^{2}-m_{f}^{2}+i \epsilon\right)} \frac{1}{\left(q_{\|}^{2}-m_{f}^{2}+i \epsilon\right)}\right],
$$

and the medium contributions containing single $(n)$ and double distribution $\left(n^{2}\right)$ functions, respectively, are also separated from eq. (3.20),

$$
\begin{aligned}
& \Pi_{n}^{\mu \nu}\left(p_{\|}\right)=-\frac{g^{2}}{2(2 \pi)^{3}} \int d k_{0} d k_{3} L^{\mu \nu}\left[\frac{n_{F}\left(k_{0}\right) \delta\left(k_{\|}^{2}-m_{f}^{2}\right)}{\left(q_{\|}^{2}-m_{f}^{2}+i \epsilon\right)}+\frac{n_{F}\left(q_{0}\right) \delta\left(q_{\|}^{2}-m_{f}^{2}\right)}{\left(k_{\|}^{2}-m_{f}^{2}+i \epsilon\right)}\right], \\
& \Pi_{n^{2}}^{\mu \nu}\left(p_{\|}\right)=-\frac{i g^{2}}{2(2 \pi)^{2}} \int d k_{0} d k_{3} L^{\mu \nu}\left[n_{F}\left(k_{0}\right) n_{F}\left(q_{0}\right) \delta\left(k_{\|}^{2}-m_{f}^{2}\right) \delta\left(q_{\|}^{2}-m_{f}^{2}\right)\right] .
\end{aligned}
$$


We have first evaluated the real-part of the vacuum contribution (3.22),

$$
\Pi_{V}^{\mu \nu}\left(p_{\|}\right)=\left(g_{\|}^{\mu \nu}-\frac{p_{\|}^{\mu} p_{\|}^{\nu}}{p_{\|}^{2}}\right) \Pi\left(p_{\|}^{2}\right),
$$

where the form factor, $\Pi\left(p_{\|}^{2}\right)$ is given by

$$
\Pi\left(p_{\|}^{2}\right)=\frac{g^{2}}{2 \pi^{3}}\left[\frac{2 m_{f}^{2}}{p_{\|}^{2}}\left(1-\frac{4 m_{f}^{2}}{p_{\|}^{2}}\right)^{-1 / 2} \ln \left\{\frac{\left(1-\frac{4 m_{f}^{2}}{p_{\|}^{2}}\right)^{1 / 2}+1}{\left(1-\frac{4 m_{f}^{2}}{p_{\|}^{2}}\right)^{1 / 2}-1}\right\}+1\right] .
$$

Hence the longitudinal $(\mu=0, \nu=0)$ component of the vacuum contribution is calculated as

$$
\Pi_{V}^{00}\left(p_{0}, p_{3}\right)=-\frac{p_{3}^{2}}{p_{\|}^{2}} \Pi\left(p_{\|}^{2}\right) .
$$

So after multiplying the transverse component (3.19) with the above longitudinal component, the vacuum part reduces to

$$
\Pi_{V}^{00}=\frac{g^{2}}{4 \pi^{2}} \sum_{f}\left|q_{f} B\right|,
$$

for the massless flavors in the static limit $\left(p_{0}=0, p_{1}, p_{2}, p_{3} \rightarrow 0\right)$. However the same vanishes for the physical quark masses in the static limit,

$$
\Pi_{V}^{00}=0 .
$$

Similarly the longitudinal component of the real part of $\Pi_{n}^{\mu \nu}\left(p_{\|}\right)$is calculated from eq. (3.23),

$$
\begin{aligned}
\Pi_{n}^{00}\left(p_{0}=0, p_{3}\right)= & -\frac{g^{2}}{2(2 \pi)^{3}} \int d k_{3}\left[\frac{L^{00}\left(k_{0}=\omega_{k}\right) n_{F}\left(k_{0}=\omega_{k}\right)}{2 \omega_{k}\left\{\omega_{k}^{2}-\omega_{q}^{2}\right\}}\right. \\
& +\frac{L^{00}\left(k_{0}=-\omega_{k}\right) n_{F}\left(k_{0}=-\omega_{k}\right)}{2 \omega_{k}\left\{\left(-\omega_{k}\right)^{2}-\omega_{q}^{2}\right\}}+\frac{L^{00}\left(k_{0}=\omega_{q}\right) n_{F}\left(k_{0}=\omega_{q}\right)}{2 \omega_{k}\left\{\omega_{q}^{2}-\omega_{k}^{2}\right\}} \\
& \left.+\frac{L^{00}\left(k_{0}=-\omega_{q}\right) n_{F}\left(k_{0}=-\omega_{q}\right)}{2 \omega_{k}\left\{\left(-\omega_{q}\right)^{2}-\omega_{k}^{2}\right\}}\right]
\end{aligned}
$$

where the different factors are defined by

$$
\begin{aligned}
L^{00} & =8\left(k_{0} q_{0}+k_{3} q_{3}+m_{f}^{2}\right), \\
\omega_{k}^{2} & =k_{3}^{2}+m_{f}^{2}, \quad \omega_{q}^{2}=q_{3}^{2}+m_{f}^{2}, \\
L^{00}\left(k_{0}=\omega_{k}\right) & =8\left(2 \omega_{k}^{2}-k_{3} p_{3}\right), \\
L^{00}\left(k_{0}=-\omega_{k}\right) & =8\left(2 \omega_{k}^{2}-k_{3} p_{3}\right), \\
L^{00}\left(k_{0}=\omega_{q}\right) & =8\left(\omega_{k}^{2}+\omega_{q}^{2}-k_{3} p_{3}\right), \\
L^{00}\left(k_{0}=-\omega_{q}\right) & =8\left(\omega_{k}^{2}+\omega_{q}^{2}-k_{3} p_{3}\right), \\
n_{F}\left(k_{0}=\omega_{k}\right) & =n_{F}\left(k_{0}=-\omega_{k}\right)=\frac{1}{e^{\beta\left|\omega_{k}\right|}+1}, \\
n_{F}\left(k_{0}=\omega_{q}\right) & =n_{F}\left(k_{0}=-\omega_{q}\right)=\frac{1}{e^{\beta\left|\omega_{q}\right|}+1} .
\end{aligned}
$$


The above contribution is further simplified into the static limit $\left(p_{0}=0\right)$ for the massless flavors,

$$
\Pi_{n}^{00}\left(p_{0}=0, p_{3}\right)=\frac{8 g^{2}}{2(2 \pi)^{3}}\left[-1+\frac{T}{p_{3}} \ln (2)-\frac{T}{p_{3}} \ln \left(1+e^{-\frac{p_{3}}{T}}\right)\right],
$$

whereas for the physical quark masses, it reduces to

$$
\begin{aligned}
\Pi_{n}^{00}\left(p_{0}=0, p_{3}\right)= & -\frac{g^{2}}{2(2 \pi)^{3}} \int d k_{3}\left[\frac{8 k_{3} n_{F}\left(\omega_{k}\right)}{\omega_{k} p_{3}}\right. \\
& \left.-\frac{8\left(k_{3}-p_{3}\right) n_{F}\left(\omega_{q}\right)}{\omega_{q} p_{3}}+\frac{16 m_{f}^{2} n_{F}\left(\omega_{k}\right)}{\omega_{k} p_{3}\left(2 k_{3}-p_{3}\right)}-\frac{16 m_{f}^{2} n_{F}\left(\omega_{q}\right)}{\omega_{q} p_{3}\left(2 k_{3}-p_{3}\right)}\right]
\end{aligned}
$$

However, the medium contribution with the square of the distribution function (3.24) is purely imaginary, so it does not contribute to the real-part of gluon self-energy,

$$
\Pi_{n^{2}}^{\mu \nu}\left(p_{\|}\right)=0
$$

Thus multiplying the transverse component (3.19) to the parallel component (3.31) we obtain the longitudinal component of the medium contribution,

$$
\Pi_{n}^{00}=-\frac{g^{2}}{4 \pi^{2}} \sum_{f}\left|q_{f} B\right|+\frac{g^{2}}{8 \pi^{2}} \sum_{f}\left|q_{f} B\right|,
$$

for the massless flavors. However, the above contribution reduces to

$$
\Pi_{n}^{00}=\frac{g^{2}}{4 \pi^{2} T} \sum_{f}\left|q_{f} B\right| \int_{0}^{\infty} d k_{3} \frac{e^{\beta \omega_{k}}}{\left(1+e^{\beta \omega_{k}}\right)^{2}}
$$

for the physical quark masses.

Finally the vacuum (3.28) and medium (3.34) contributions are added together to give the longitudinal component of the real part of the gluon self-energy,

$$
\Pi^{00}=\frac{g^{2}}{8 \pi^{2}} \sum_{f}\left|q_{f} B\right|,
$$

for the massless quarks. Similarly for the physical quark masses, the real part gives rise to

$$
\Pi^{00}=\frac{g^{2}}{4 \pi^{2} T} \sum_{f}\left|q_{f} B\right| \int_{0}^{\infty} d k_{3} \frac{e^{\beta \omega_{k}}}{\left(1+e^{\beta \omega_{k}}\right)^{2}} .
$$

Therefore the longitudinal component (3.36) gives the Debye mass (3.16) for the massless quarks,

$$
m_{D}^{2}=\frac{g^{2}}{8 \pi^{2}} \sum_{f}\left|q_{f} B\right|,
$$

which was recently obtained by one of us [64] and also by others using the different approaches $[33,65]$. Thus the Debye mass in the presence of a strong magnetic field depends 
solely on the magnetic field and is independent of the temperature, hence the collective behavior of the medium is strongly affected by the magnetic field.

However, for the physical quark masses, the Debye mass is obtained as

$$
m_{D}^{2}=\frac{g^{2}}{4 \pi^{2} T} \sum_{f}\left|q_{f} B\right| \int_{0}^{\infty} d k_{3} \frac{e^{\beta \sqrt{k_{3}^{2}+m_{f}^{2}}}}{\left(1+e^{\beta \sqrt{k_{3}^{2}+m_{f}^{2}}}\right)^{2}},
$$

which, in principle, depends on both the magnetic field and the temperature. However the dependence on the temperature is too much weak, which in fact becomes independent of temperature beyond a certain temperature.

\section{Thermodynamic observables in the strong magnetic field}

\subsection{Free energy and pressure}

The free energy of a hot QCD medium is given by the sum of free energies due to quarks $\left(\mathcal{F}_{q}\right)$ and gluons $\left(\mathcal{F}_{g}\right)$, which are obtained by the functional determinant of the effective quark and gluon propagators, respectively. Thereafter the pressure is obtained by the negative of the free energy in the thermodynamic limit. So we are going to calculate the free energies and the pressures due to quarks and gluons.

\subsubsection{Quark contribution}

The free energy due to $N_{f}$ quarks with $N_{c}$ colors is obtained by the effective quark propagator, $S(P)$ from eq. (2.1),

$$
\begin{aligned}
\mathcal{F}_{q} & =N_{c} N_{f} \int \frac{d^{4} P}{(2 \pi)^{4}} \ln [\operatorname{det}(S(P))] \\
& =-N_{c} N_{f} \int \frac{d^{4} P}{(2 \pi)^{4}} \ln \left[\operatorname{det}\left(\gamma^{\|} \cdot p_{\|}-m_{f}-\Sigma\left(p_{\|}\right)\right)\right] .
\end{aligned}
$$

In the presence of an external magnetic field in the $z$ direction, the momentum integration is again factorized into the momentum parallel and perpendicular to the magnetic field. By the same reason, the integrand, which involves the quark self-energy, depends only on the longitudinal momentum in SMF limit.

For the sake of simplicity, we rewrite the quark self-energy (2.61) in terms of the constant and the momentum-dependent terms,

$$
\begin{aligned}
\Sigma\left(p_{\|}\right) & =J_{+}\left(\gamma^{\|} \cdot p_{\|}\right) C+J_{+} D+E+F, \text { with } \\
C & =\frac{g^{2}}{6 \pi^{2}}\left[-\frac{1}{2}-\frac{\left|q_{f} B\right|}{2 m_{f}^{2}}\left\{\ln \left(\frac{\left|q_{f} B\right|}{m_{f}^{2}}\right)-1\right\}\right], \\
D & =\frac{g^{2}}{6 \pi^{2}}\left[\frac{\left|q_{f} B\right|}{m_{f}}\left\{\ln \left(\frac{\left|q_{f} B\right|}{m_{f}^{2}}\right)-1\right\}\right], \\
E & =\frac{g^{2} m_{f}}{3 \pi^{2}}, \\
F & =-\frac{2 g^{2} m_{f}}{3 \pi^{2}} \ln \left(\frac{\left|q_{f} B\right|}{\left(p_{\|}-m_{f}\right)^{2}}\right)\left[\ln \left(\frac{m_{f}}{\pi T}\right)+\gamma_{E}\right],
\end{aligned}
$$


and then evaluate the determinant,

$$
\begin{aligned}
\operatorname{det}\left[\gamma^{\|} \cdot p_{\|}-m_{f}-\Sigma\left(p_{\|}\right)\right]= & {\left[p_{\|}^{2}(1-2 C)^{2}-\left(m_{f}+2 D+E+F\right)^{2}\right] } \\
& \times\left[p_{\|}^{2}-\left(m_{f}+E+F\right)^{2}\right] .
\end{aligned}
$$

Thus after plugging the determinant into the integration (4.1), the quark contribution is expressed as

$$
\begin{aligned}
\mathcal{F}_{q}=-N_{c} N_{f} \int \frac{d^{2} p_{\perp}}{(2 \pi)^{2}} \int \frac{d^{2} p_{\|}}{(2 \pi)^{2}} \ln \left[\left\{p_{\|}^{2}(1-2 C)^{2}-\left(m_{f}+2 D+E+F\right)^{2}\right\}\right. \\
\left.\quad \times\left\{p_{\|}^{2}-\left(m_{f}+E+F\right)^{2}\right\}\right] \\
=-2 N_{c} N_{f} \int \frac{d^{2} p_{\perp}}{(2 \pi)^{2}} \int \frac{d^{2} p_{\|}}{(2 \pi)^{2}} \ln \left(p_{\|}^{2}\right) \\
-2 N_{c} N_{f} \int \frac{d^{2} p_{\perp}}{(2 \pi)^{2}} \frac{1}{2} \int \frac{d^{2} p_{\|}}{(2 \pi)^{2}} \ln \left[\left\{(1-2 C)^{2}-\frac{1}{p_{\|}^{2}}\left(m_{f}+2 D+E+F\right)^{2}\right\}\right. \\
\equiv-\frac{N_{c} N_{f}\left|q_{f} B\right|}{2 \pi}\left(I_{1 p_{\|}}+I_{2 p_{\|}}\right) .
\end{aligned}
$$

At finite temperature, the first integral, $I_{1 p_{\|}}$has been frequently solved in the imaginarytime formalism, where the energy integrals are replaced by the discrete Matsubara frequency sums, i.e. $\int \frac{d p_{0}}{2 \pi} \rightarrow T \sum_{n}$. For quarks, the discrete frequencies $\left(\omega_{n}\right)$ are $(2 n+1) \pi T$, where $n=0,1,2, \cdots$. Thus we solve the integral, $I_{1 p_{\|}}$analytically as

$$
\begin{aligned}
I_{1 p_{\|}} & =\int \frac{d p_{0}}{2 \pi} \int \frac{d p_{3}}{2 \pi} \ln \left(p_{0}^{2}-p_{3}^{2}\right) \\
& =\frac{\pi T^{2}}{6} .
\end{aligned}
$$

The second integral, $I_{2 p_{\|}}$is rewritten as

$$
\begin{aligned}
& I_{2 p_{\|}}=\frac{1}{2} \int \frac{d^{2} p_{\|}}{(2 \pi)^{2}} \ln \left[\left\{(1-2 C)^{2}-\frac{1}{p_{\|}^{2}}\left(m_{f}+2 D+E+F\right)^{2}\right\}\right. \\
& \left.\times\left\{1-\frac{1}{p_{\|}^{2}}\left(m_{f}+E+F\right)^{2}\right\}\right] \\
& =\int_{0}^{\left|q_{f} B\right|} \frac{d p_{\|}^{2}}{8 \pi} \ln \left[\left\{(1-2 C)^{2}-\frac{1}{p_{\|}^{2}}\left[m_{f}+2 D+E+W \ln \left(\frac{\left|q_{f} B\right|}{\left(p_{\|}-m_{f}\right)^{2}}\right)\right]^{2}\right\}\right. \\
& \left.\times\left\{1-\frac{1}{p_{\|}^{2}}\left[m_{f}+E+W \ln \left(\frac{\left|q_{f} B\right|}{\left(p_{\|}-m_{f}\right)^{2}}\right)\right]^{2}\right\}\right],
\end{aligned}
$$


by reexpressing the momentum-dependent term, $F$ as

$$
\begin{gathered}
F=W \ln \left(\frac{\left|q_{f} B\right|}{\left(p_{\|}-m_{f}\right)^{2}}\right), \text { with } \\
W=-\frac{2 g^{2} m_{f}}{3 \pi^{2}}\left\{\ln \left(\frac{m_{f}}{\pi T}\right)+\gamma_{E}\right\} .
\end{gathered}
$$

Now, substituting the integrals $I_{1 p_{\|}}$and $I_{2 p_{\|}}$in eq. (4.8), the free energy due to the quark contribution in a strong magnetic field is obtained as

$$
\begin{aligned}
\mathcal{F}_{q}= & -\frac{N_{c} N_{f}\left|q_{f} B\right|}{4}\left[\frac{T^{2}}{3}+\int_{0}^{\left|q_{f} B\right|} \frac{d p_{\|}^{2}}{4 \pi^{2}} \ln \left[\left\{(1-2 C)^{2}\right.\right.\right. \\
& \left.-\frac{1}{p_{\|}^{2}}\left[m_{f}+2 D+E+W \ln \left(\frac{\left|q_{f} B\right|}{\left(p_{\|}-m_{f}\right)^{2}}\right)\right]^{2}\right\} \\
& \left.\times\left\{1-\frac{1}{p_{\|}^{2}}\left[m_{f}+E+W \ln \left(\frac{\left|q_{f} B\right|}{\left(p_{\|}-m_{f}\right)^{2}}\right)\right]^{2}\right\}\right] .
\end{aligned}
$$

Hence the negative of the above free energy in the thermodynamic limit gives the pressure. Thus substituting the expressions for $C, D, E$ and $W$, we get the quark contribution,

$$
\begin{aligned}
P_{q}= & \frac{N_{c} N_{f}\left|q_{f} B\right|}{4}\left[\frac{T^{2}}{3}+\int_{0}^{\left|q_{f} B\right|} \frac{d p_{\|}^{2}}{4 \pi^{2}} \ln \left[\left\{\left(1-\frac{g^{2}}{3 \pi^{2}}\left[-\frac{1}{2}\right.\right.\right.\right.\right. \\
& \left.\left.-\frac{\left|q_{f} B\right|}{2 m_{f}^{2}}\left\{\ln \left(\frac{\left|q_{f} B\right|}{m_{f}^{2}}\right)-1\right\}\right]\right)^{2} \\
& -\frac{1}{p_{\|}^{2}}\left[m_{f}+\frac{g^{2}}{3 \pi^{2}}\left[m_{f}+\frac{\left|q_{f} B\right|}{m_{f}}\left\{\ln \left(\frac{\left|q_{f} B\right|}{m_{f}^{2}}\right)-1\right\}\right]\right. \\
& \left.\left.-\frac{2 g^{2} m_{f}}{3 \pi^{2}}\left\{\ln \left(\frac{m_{f}}{\pi T}\right)+\gamma_{E}\right\} \ln \left(\frac{\left|q_{f} B\right|}{\left(p_{\|}-m_{f}\right)^{2}}\right)\right]^{2}\right\}\left\{1-\frac{1}{p_{\|}^{2}}\left[m_{f}+\frac{g^{2} m_{f}}{3 \pi^{2}}\right.\right. \\
& \left.\left.\left.-\frac{2 g^{2} m_{f}}{3 \pi^{2}}\left\{\ln \left(\frac{m_{f}}{\pi T}\right)+\gamma_{E}\right\} \ln \left(\frac{\left|q_{f} B\right|}{\left(p_{\|}-m_{f}\right)^{2}}\right)\right]^{2}\right\}\right] .
\end{aligned}
$$

\subsubsection{Gluon contribution}

The free energy due to the gluons in adjoint representation of $\mathrm{SU}\left(N_{c}\right)$ gauge theory is given by both the (two) transverse and longitudinal modes,

$$
\begin{aligned}
\mathcal{F}_{g} & =\left(N_{c}^{2}-1\right)\left[2 \mathcal{F}_{g}^{T}+\mathcal{F}_{g}^{L}\right] \\
& =\left(N_{c}^{2}-1\right)\left[\int \frac{d^{4} P}{(2 \pi)^{4}} \ln \left[-\Delta_{T}(P)\right]^{-1}+\frac{1}{2} \int \frac{d^{4} P}{(2 \pi)^{4}} \ln \left[\Delta_{L}(P)\right]^{-1}\right] \\
& =-\left(N_{c}^{2}-1\right)\left[\int \frac{d^{4} P}{(2 \pi)^{4}} \ln \left[-\Delta_{T}(P)\right]+\frac{1}{2} \int \frac{d^{4} P}{(2 \pi)^{4}} \ln \left[\Delta_{L}(P)\right]\right] .
\end{aligned}
$$


After substituting the values of $\Delta_{T}(P)$ and $\Delta_{L}(P)$ from equations (3.14) and (3.15), respectively, we obtain the gluon contribution,

$$
\mathcal{F}_{g}=-\left(N_{c}^{2}-1\right)\left[\int \frac{d^{4} P}{(2 \pi)^{4}} \ln \left(\frac{1}{P^{2}+\Pi_{T}(P)}\right)+\frac{1}{2} \int \frac{d^{4} P}{(2 \pi)^{4}} \ln \left(\frac{1}{\mathbf{p}^{2}+\Pi_{L}(P)}\right)\right],
$$

where the dependence of the magnetic field enters into the $\Pi_{T}$ and $\Pi_{L}$ through the Debye mass (3.39).

For the gluons in a thermal medium, the loop momenta may be hard (i.e. order of $T$ ) or soft (i.e. order of $g T$ ). In imaginary-time formalism, the gluon energy $(2 n \pi T)$ is an integer multiple of $2 \pi T$, so the soft-momentum regime requires $n=0$. On the other hand, for the quarks, the discrete energies $((2 n+1) \pi T)$ can never be zero even for $n=0$, so the quark loop is always hard. However, the gluons are not affected by the presence of a magnetic field, so the highest scale for them in a medium is still the temperature. Thus the hard gauge field fluctuations produce a small perturbation on the motion of a plasma particle. However, this is not so for an excitation at the soft loop-momenta which is nonperturbatively renormalized by the hard thermal fluctuations. The free energy due to the gluon contribution is therefore calculated up to $\mathcal{O}\left(g^{4}\right)$ from both the hard and soft scales in [51],

$$
\begin{aligned}
\mathcal{F}_{g}=-\left(N_{c}^{2}-1\right)[ & \frac{\pi^{2} T^{4}}{45}-\frac{T^{2} m_{D}^{2}}{24}+\frac{T m_{D}^{3}}{12 \pi} \\
& \left.+\frac{m_{D}^{4}}{128 \pi^{2}}\left\{\frac{1}{\epsilon}+2 \ln \left(\frac{\Lambda}{4 \pi T}\right)-7+2 \gamma_{E}+\frac{2 \pi^{2}}{3}\right\}\right],
\end{aligned}
$$

where $\Lambda$ is the renormalization scale and the divergent term $(1 / \epsilon)$ is isolated by the dimensional regularization, which needs to be compensated by the vacuum counter-term,

$$
\Delta \mathcal{E}_{0}=\frac{N_{c}^{2}-1}{128 \pi^{2} \epsilon} m_{D}^{4}
$$

Thus the above counter-term gives the renormalized gluon contribution to the free energy and the negative of this free energy determines the QCD pressure as

$$
P_{g}=\left(N_{c}^{2}-1\right)\left[\frac{\pi^{2} T^{4}}{45}-\frac{T^{2} m_{D}^{2}}{24}+\frac{T m_{D}^{3}}{12 \pi}+\frac{m_{D}^{4}}{128 \pi^{2}}\left\{2 \ln \left(\frac{\Lambda}{4 \pi T}\right)-7+2 \gamma_{E}+\frac{2 \pi^{2}}{3}\right\}\right] .
$$

After plugging the Debye mass (3.38) for two massless $u$ and $d$ flavors, the explicit dependence of the magnetic field will be seen in the gluon contribution of the pressure,

$$
\begin{aligned}
P_{g}=\left(N_{c}^{2}-1\right)[ & \frac{\pi^{2} T^{4}}{45}-g^{2} \frac{T^{2} e B}{192 \pi^{2}}+g^{3} \frac{T(e B)^{\frac{3}{2}}}{192 \sqrt{2} \pi^{4}} \\
& \left.+g^{4} \frac{(e B)^{2}}{8192 \pi^{6}}\left\{2 \ln \left(\frac{\Lambda}{4 \pi T}\right)-7+2 \gamma_{E}+\frac{2 \pi^{2}}{3}\right\}\right] .
\end{aligned}
$$




\subsubsection{Total pressure}

The total one-loop pressure of the strongly magnetized hot QCD matter is thus obtained by adding both the quark and gluon contributions,

$$
\begin{aligned}
P(T, e B)= & \frac{N_{c} N_{f}\left|q_{f} B\right|}{4}\left[\frac{T^{2}}{3}+\int_{0}^{\left|q_{f} B\right|} \frac{d p_{\|}^{2}}{4 \pi^{2}} \ln \left[\left\{\left(1-\frac{g^{2}}{3 \pi^{2}}\left[-\frac{1}{2}\right.\right.\right.\right.\right. \\
& \left.\left.-\frac{\left|q_{f} B\right|}{2 m_{f}^{2}}\left\{\ln \left(\frac{\left|q_{f} B\right|}{m_{f}^{2}}\right)-1\right\}\right]\right)^{2} \\
& -\frac{1}{p_{\|}^{2}}\left[m_{f}+\frac{g^{2}}{3 \pi^{2}}\left[m_{f}+\frac{\left|q_{f} B\right|}{m_{f}}\left\{\ln \left(\frac{\left|q_{f} B\right|}{m_{f}^{2}}\right)-1\right\}\right]\right. \\
& \left.\left.-\frac{2 g^{2} m_{f}}{3 \pi^{2}}\left\{\ln \left(\frac{m_{f}}{\pi T}\right)+\gamma_{E}\right\} \ln \left(\frac{\left|q_{f} B\right|}{\left(p_{\|}-m_{f}\right)^{2}}\right)\right]^{2}\right\}\left\{1-\frac{1}{p_{\|}^{2}}\left[m_{f}+\frac{g^{2} m_{f}}{3 \pi^{2}}\right.\right. \\
& \left.\left.\left.-\frac{2 g^{2} m_{f}}{3 \pi^{2}}\left\{\ln \left(\frac{m_{f}}{\pi T}\right)+\gamma_{E}\right\} \ln \left(\frac{\left|q_{f} B\right|}{\left(p_{\|}-m_{f}\right)^{2}}\right)\right]^{2}\right\}\right] \\
& +\left(N_{c}^{2}-1\right)\left[\frac{\pi^{2} T^{4}}{45}-\frac{T^{2} m_{D}^{2}}{24}+\frac{T m_{D}^{3}}{12 \pi}\right. \\
& \left.+\frac{m_{D}^{4}}{128 \pi^{2}}\left\{2 \ln \left(\frac{\Lambda}{4 \pi T}\right)-7+2 \gamma_{E}+\frac{2 \pi^{2}}{3}\right\}\right]
\end{aligned}
$$

where the renormalization scale $\Lambda$ is set at $2 \pi T$ in the pure thermal medium. The ideal component can thus be read as

$$
P_{\text {ideal }}(T, e B)=N_{c} N_{f} \frac{\left|q_{f} B\right| T^{2}}{12}+\left(N_{c}^{2}-1\right) \frac{\pi^{2} T^{4}}{45} .
$$

Before calculating the thermodynamic observables, we should be careful in choosing the range of temperatures and magnetic fields, which should be compatible to the SMF limit. For example, while computing the pressure as a function of the magnetic field at a temperature $T=300 \mathrm{MeV}$, the starting value of the magnetic field has to be greater than $\sim 4.6 m_{\pi}^{2}$, however, we have taken the starting magnetic field, $e B=10 m_{\pi}^{2}$, which is almost twice the above marginal value. Similarly for calculating the pressure as a function of temperature up to $T=400 \mathrm{MeV}$, we have fixed the magnetic fields at $e B=15 m_{\pi}^{2}, 25 m_{\pi}^{2}$, and $50 m_{\pi}^{2}$. However, we have studied the variations of the thermodynamic observables with either the magnetic field or with the temperature in $\mathrm{GeV}$, with the conversion factor, $m_{\pi}^{2} \sim 0.02 \mathrm{GeV}^{2}$.

To see how the pressure of a hot QCD matter with two light flavors is affected in the presence of a strong magnetic field, we have shown the variation of pressure as a function of the magnetic field ${ }^{1}$ at a fixed temperature of the medium and vice versa in figures $3 \mathrm{a}$ and $3 \mathrm{~b}$, respectively. We have noticed that the pressure of a thermal medium in the

\footnotetext{
${ }^{1}$ To compare the rate of increase of the pressure with respect to the temperature with the rate of increase of the pressure with respect to the magnetic field at the same dimension, we have computed the pressure as a function of the square root of the magnetic field $(\sqrt{e B})$.
} 


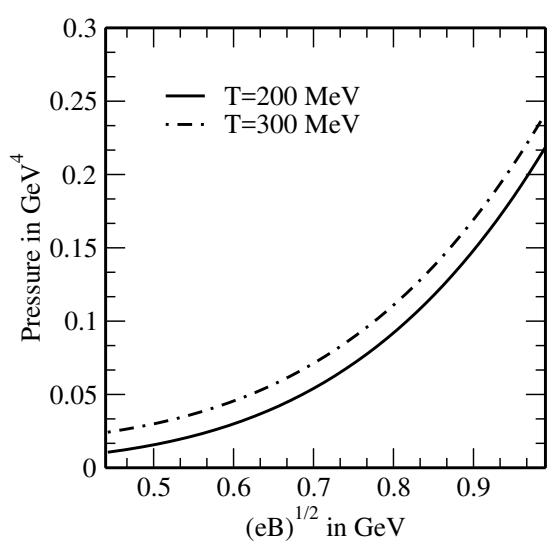

(a)

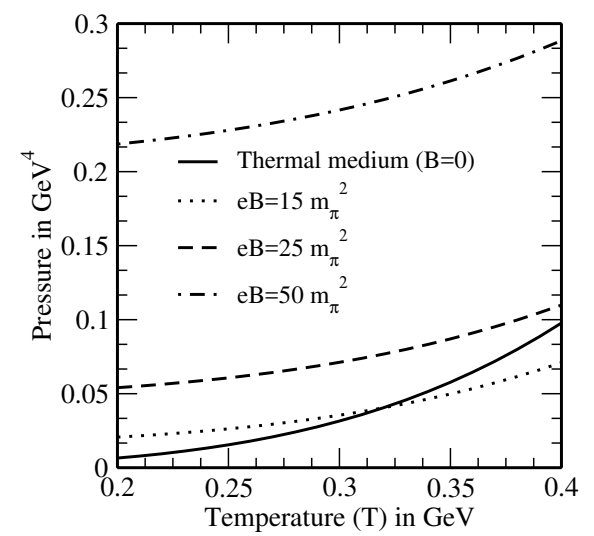

(b)

Figure 3. The variation of pressure of a hot QCD medium for two light flavors as a function of $\sqrt{e B}$ at fixed temperatures (a) and as a function of $T$ at different strong magnetic fields (b).

presence of a strong magnetic field increases with the magnetic field faster than with the temperature. The above contrast in the behavior reflects the fact that the dominant scale of a thermal medium in SMF limit $\left(e B \gg T^{2}\right)$ is the magnetic field (precisely $\sqrt{e B}$ ), not the temperature usually assumed for a thermal medium in the absence of a magnetic field, so the pressure is more sensitive to the scale associated with the magnetic field, not the temperature. In addition, the presence of a strong magnetic field makes the pressure of the thermal QCD medium larger than the thermal medium in the absence of a magnetic field (denoted by the solid line in figure $3 \mathrm{~b}$ ). These vital observations will facilitate to understand the effects of the magnetic field on the entropy density (in figure 5).

Recently the thermodynamic pressure in a magnetic field has been studied in the lattice QCD with $(2+1)$ flavors [36], where they have also noticed the similar increase in the (longitudinal) pressure with the magnetic field. To see how the pressure of the interacting quarks and gluons in a hot QCD medium approaches to the noninteracting (ideal) limit asymptotically, we have computed the pressure in units of the ideal pressure (4.22) as functions of both the magnetic field and the temperature in figures $4 \mathrm{a}$ and $4 \mathrm{~b}$, respectively. In figure $4 \mathrm{a}$, it is found that the deviation of the pressure from its ideal value increases with the magnetic field, implying that the pressure of a thermal QCD medium never achieves its ideal limit asymptotically. On the other hand, the pressure at a fixed magnetic field (in figure $4 \mathrm{~b}$ ) approaches to its ideal limit with the temperature. It may be remembered that in the SMF limit, one cannot arbitrarily increase the temperature due to its constraint $\left(e B \gg T^{2}\right)$.

\subsection{Entropy density}

To see how the available number of microstates to a given macrostate of a thermal QCD medium are affected in the presence of a strong magnetic field, we will now calculate the entropy density by partially differentiating the pressure (4.21) with respect to the temperature,

$$
\begin{aligned}
S & =\frac{\partial P}{\partial T} \\
& \equiv S_{q}+S_{g},
\end{aligned}
$$




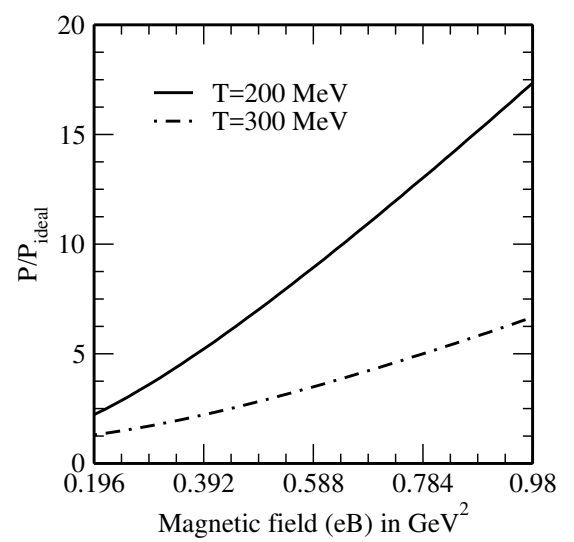

(a)

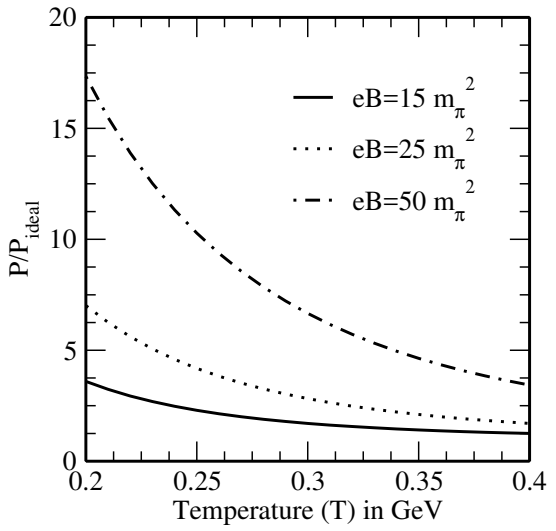

(b)

Figure 4. The variation of QCD pressure normalized by its ideal value as a function of magnetic field at different temperatures (a) and as a function of temperature at different strong magnetic fields (b).

where the quark contribution $\left(S_{q}\right)$ is calculated as

$$
\begin{aligned}
S_{q}= & \frac{N_{c} N_{f}\left|q_{f} B\right|}{6}\left[T-\frac{m_{f} g^{2}}{2 T \pi^{4}} \int_{0}^{\left|q_{f} B\right|} d p_{\|}^{2} \frac{1}{p_{\|}^{2}} \ln \left(\frac{\left|q_{f} B\right|}{\left(p_{\|}-m_{f}\right)^{2}}\right)\right. \\
& \times\left\{\left[m_{f}+\frac{g^{2}}{3 \pi^{2}}\left(m_{f}+\frac{\left|q_{f} B\right|}{m_{f}}\left\{\ln \left(\frac{\left|q_{f} B\right|}{m_{f}^{2}}\right)-1\right\}\right)\right.\right. \\
& \left.-\frac{2 g^{2} m_{f}}{3 \pi^{2}}\left\{\ln \left(\frac{m_{f}}{\pi T}\right)+\gamma_{E}\right\} \ln \left(\frac{\left|q_{f} B\right|}{\left(p_{\|}-m_{f}\right)^{2}}\right)\right] \\
& /\left[\left\{1-\frac{g^{2}}{3 \pi^{2}}\left(-\frac{1}{2}-\frac{\left|q_{f} B\right|}{2 m_{f}^{2}}\left\{\ln \left(\frac{\left|q_{f} B\right|}{m_{f}^{2}}\right)-1\right\}\right)\right\}^{2}\right. \\
& -\frac{1}{p_{\|}^{2}}\left\{m_{f}+\frac{g^{2}}{3 \pi^{2}}\left(m_{f}+\frac{\left|q_{f} B\right|}{m_{f}}\left\{\ln \left(\frac{\left|q_{f} B\right|}{m_{f}^{2}}\right)-1\right\}\right)\right. \\
& \left.\left.-\frac{2 g^{2} m_{f}}{3 \pi^{2}}\left\{\ln \left(\frac{m_{f}}{\pi T}\right)+\gamma_{E}\right\} \ln \left(\frac{\left|q_{f} B\right|}{\left(p_{\|}-m_{f}\right)^{2}}\right)\right\}^{2}\right]+\left[m_{f}+\frac{g^{2} m_{f}}{3 \pi^{2}}\right. \\
& \left.-\frac{2 g^{2} m_{f}}{3 \pi^{2}}\left\{\ln \left(\frac{m_{f}}{\pi T}\right)+\gamma_{E}\right\} \ln \left(\frac{\left|q_{f} B\right|}{\left(p_{\|}-m_{f}\right)^{2}}\right)\right] /\left[1-\frac{1}{p_{\|}^{2}}\left\{m_{f}+\frac{g^{2} m_{f}}{3 \pi^{2}}\right.\right. \\
& \left.\left.\left.\left.-\frac{2 g^{2} m_{f}}{3 \pi^{2}}\left\{\ln \left(\frac{m_{f}}{\pi T}\right)+\gamma_{E}\right\} \ln \left(\frac{\left|q_{f} B\right|}{\left(p_{\|}-m_{f}\right)^{2}}\right)\right\}^{2}\right]\right\}\right]
\end{aligned}
$$

Similarly, the gluon contribution is calculated from the gluon pressure (4.19),

$$
S_{g}=\left(N_{c}^{2}-1\right)\left[\frac{4 \pi^{2} T^{3}}{45}-\frac{T m_{D}^{2}}{12}+\frac{m_{D}^{3}}{12 \pi}\right]
$$




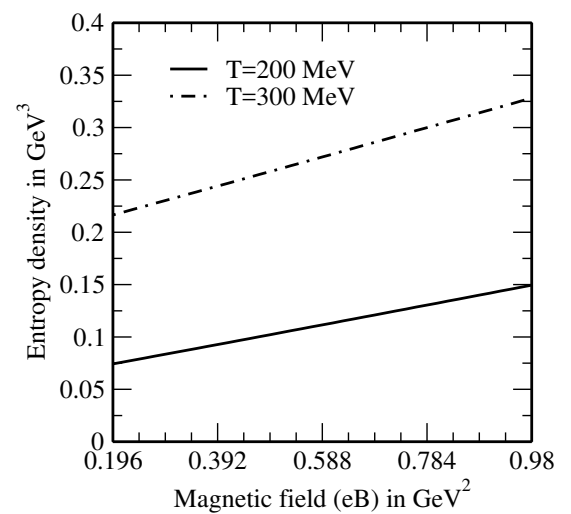

(a)

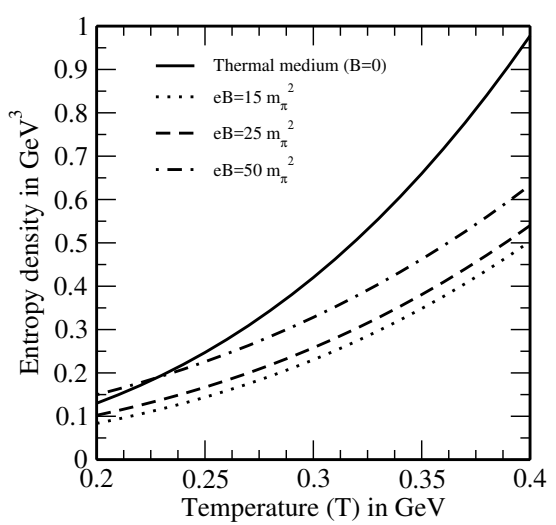

(b)

Figure 5. The variation of entropy density as a function of magnetic field at different temperatures (a) and as a function of temperature at different strong magnetic fields (b).

In addition to the temperature, the explicit dependence of the magnetic field can be found out by substituting the Debye mass for the two massless flavors from eq. (3.38) into the above equation.

$$
S_{g}=\left(N_{c}^{2}-1\right)\left[\frac{4 \pi^{2} T^{3}}{45}-g^{2} \frac{T e B}{96 \pi^{2}}+g^{3} \frac{(e B)^{\frac{3}{2}}}{192 \sqrt{2} \pi^{4}}\right] .
$$

However, we could substitute the Debye mass for two light flavors (3.39) to visualize the magnetic field dependence. In that case, the partial temperature derivative of the (square) Debye mass (3.39) can be approximately taken to zero for the simplification of calculation because albeit the (square) Debye mass depends on both the temperature and the magnetic field but the temperature-dependence is too weak to consider its temperature derivative.

In figure $5 \mathrm{a}$, we have shown how the entropy densities of a hot QCD medium at fixed temperatures $(T=200 \mathrm{MeV}$ and $T=300 \mathrm{MeV})$ have been affected by the increase of the magnetic fields and found that the entropy density for a given medium increases almost linearly with the magnetic field. However, for the higher temperature, the increase becomes a little bit faster. Similarly the variation of the entropy density with the temperature in SMF backgrounds, $e B=15 \mathrm{~m}_{\pi}^{2}, 25 \mathrm{~m}_{\pi}^{2}$, and $50 \mathrm{~m}_{\pi}^{2}$ is displayed in figure $5 \mathrm{~b}$. Here we have noticed that the entropy density in the presence of a strong magnetic field not only becomes smaller but its increase becomes slower compared to the thermal medium in the absence of a magnetic field (denoted by the solid line, $B=0$ ). The above observation can be understood qualitatively: the strong magnetic field restricts the dynamics of quarks originally in the four dimensions to the two dimensions, so the phase-space gets squeezed to the two dimensions. Since the number of microstates is proportional to the available phase space, hence the entropy density of a thermal medium decreases, which corroborates the smaller slope observed in the variation of the pressure with the temperature due to the presence of strong magnetic field in figure $3 \mathrm{~b}$. 


\subsection{Energy density}

We are now in a position to calculate the energy density for a baryonless $\left(\mu_{q}=0\right)$ hot QCD medium in a strong magnetic field from the following thermodynamic relation,

$$
\begin{aligned}
\varepsilon & =-P+T S \\
& \equiv \varepsilon_{q}+\varepsilon_{g},
\end{aligned}
$$

where the quark contribution is calculated as

$$
\begin{aligned}
& \varepsilon_{q}=-P_{q}+T S_{q} \\
& =\frac{N_{c} N_{f}\left|q_{f} B\right|}{6}\left[\frac{T^{2}}{2}-\frac{3}{8 \pi^{2}} \int_{0}^{\left|q_{f} B\right|} d p_{\|}^{2} \ln \left[\left\{\left(1-\frac{g^{2}}{3 \pi^{2}}\left[-\frac{1}{2}\right.\right.\right.\right.\right. \\
& \left.\left.-\frac{\left|q_{f} B\right|}{2 m_{f}^{2}}\left\{\ln \left(\frac{\left|q_{f} B\right|}{m_{f}^{2}}\right)-1\right\}\right]\right)^{2} \\
& -\frac{1}{p_{\|}^{2}}\left[m_{f}+\frac{g^{2}}{3 \pi^{2}}\left[m_{f}+\frac{\left|q_{f} B\right|}{m_{f}}\left\{\ln \left(\frac{\left|q_{f} B\right|}{m_{f}^{2}}\right)-1\right\}\right]\right. \\
& \left.\left.-\frac{2 g^{2} m_{f}}{3 \pi^{2}}\left\{\ln \left(\frac{m_{f}}{\pi T}\right)+\gamma_{E}\right\} \ln \left(\frac{\left|q_{f} B\right|}{\left(p_{\|}-m_{f}\right)^{2}}\right)\right]^{2}\right\}\left\{1-\frac{1}{p_{\|}^{2}}\left[m_{f}+\frac{g^{2} m_{f}}{3 \pi^{2}}\right.\right. \\
& \left.\left.\left.-\frac{2 g^{2} m_{f}}{3 \pi^{2}}\left\{\ln \left(\frac{m_{f}}{\pi T}\right)+\gamma_{E}\right\} \ln \left(\frac{\left|q_{f} B\right|}{\left(p_{\|}-m_{f}\right)^{2}}\right)\right]^{2}\right\}\right] \\
& -\frac{m_{f} g^{2}}{2 \pi^{4}} \int_{0}^{\left|q_{f} B\right|} d p_{\|}^{2} \frac{1}{p_{\|}^{2}} \ln \left(\frac{\left|q_{f} B\right|}{\left(p_{\|}-m_{f}\right)^{2}}\right) \\
& \times\left\{\left[m_{f}+\frac{g^{2}}{3 \pi^{2}}\left(m_{f}+\frac{\left|q_{f} B\right|}{m_{f}}\left\{\ln \left(\frac{\left|q_{f} B\right|}{m_{f}^{2}}\right)-1\right\}\right)\right.\right. \\
& \left.-\frac{2 g^{2} m_{f}}{3 \pi^{2}}\left\{\ln \left(\frac{m_{f}}{\pi T}\right)+\gamma_{E}\right\} \ln \left(\frac{\left|q_{f} B\right|}{\left(p_{\|}-m_{f}\right)^{2}}\right)\right] \\
& /\left[\left\{1-\frac{g^{2}}{3 \pi^{2}}\left(-\frac{1}{2}-\frac{\left|q_{f} B\right|}{2 m_{f}^{2}}\left\{\ln \left(\frac{\left|q_{f} B\right|}{m_{f}^{2}}\right)-1\right\}\right)\right\}^{2}\right. \\
& -\frac{1}{p_{\|}^{2}}\left\{m_{f}+\frac{g^{2}}{3 \pi^{2}}\left(m_{f}+\frac{\left|q_{f} B\right|}{m_{f}}\left\{\ln \left(\frac{\left|q_{f} B\right|}{m_{f}^{2}}\right)-1\right\}\right)\right. \\
& \left.\left.-\frac{2 g^{2} m_{f}}{3 \pi^{2}}\left\{\ln \left(\frac{m_{f}}{\pi T}\right)+\gamma_{E}\right\} \ln \left(\frac{\left|q_{f} B\right|}{\left(p_{\|}-m_{f}\right)^{2}}\right)\right\}^{2}\right]+\left[m_{f}+\frac{g^{2} m_{f}}{3 \pi^{2}}\right. \\
& \left.-\frac{2 g^{2} m_{f}}{3 \pi^{2}}\left\{\ln \left(\frac{m_{f}}{\pi T}\right)+\gamma_{E}\right\} \ln \left(\frac{\left|q_{f} B\right|}{\left(p_{\|}-m_{f}\right)^{2}}\right)\right] /\left[1-\frac{1}{p_{\|}^{2}}\left\{m_{f}+\frac{g^{2} m_{f}}{3 \pi^{2}}\right.\right. \\
& \left.\left.\left.\left.-\frac{2 g^{2} m_{f}}{3 \pi^{2}}\left\{\ln \left(\frac{m_{f}}{\pi T}\right)+\gamma_{E}\right\} \ln \left(\frac{\left|q_{f} B\right|}{\left(p_{\|}-m_{f}\right)^{2}}\right)\right\}^{2}\right]\right\}\right]
\end{aligned}
$$




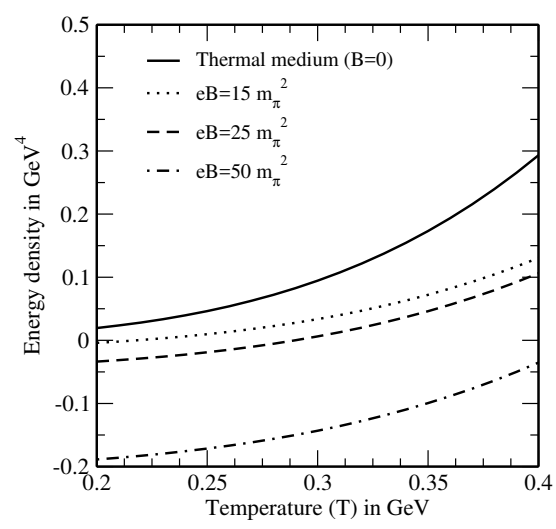

Figure 6. The variation of energy density with temperature at different magnetic fields.

and the gluon contribution is given by

$$
\begin{aligned}
\varepsilon_{g} & =-P_{g}+T S_{g} \\
& =\left(N_{c}^{2}-1\right)\left[\frac{\pi^{2} T^{4}}{15}-\frac{T^{2} m_{D}^{2}}{24}-\frac{m_{D}^{4}}{128 \pi^{2}}\left\{2 \ln \left(\frac{\Lambda}{4 \pi T}\right)-7+2 \gamma_{E}+\frac{2 \pi^{2}}{3}\right\}\right] .
\end{aligned}
$$

The dependence of the magnetic field can be seen explicitly by replacing the Debye mass for two massless flavors from eq. (3.38),

$$
\varepsilon_{g}=\left(N_{c}^{2}-1\right)\left[\frac{\pi^{2} T^{4}}{15}-g^{2} \frac{T^{2} e B}{192 \pi^{2}}-g^{4} \frac{(e B)^{2}}{8192 \pi^{6}}\left\{2 \ln \left(\frac{\Lambda}{4 \pi T}\right)-7+2 \gamma_{E}+\frac{2 \pi^{2}}{3}\right\}\right] .
$$

To see how the energy density of a thermal medium gets affected by an external strong magnetic field, we have computed the energy density as a function of temperature at different strengths of the magnetic fields in figure 6 . We have found that the energy density of the medium becomes smaller due to the presence of the magnetic field and it increases very slowly with the temperature compared to the thermal medium in the absence of a magnetic field $(\mathrm{B}=0$, denoted by the solid line). This observation reconciles with the observation on the variation of the entropy density with the temperature (in figure $5 \mathrm{~b}$ ). Therefore the decrease of the entropy density attributes to the reduction of the phase space, which is an artifact of the strong magnetic field.

\subsection{Speed of sound}

The square of the speed of sound $\left(c_{s}^{2}\right)$ relates the pressure with the energy density $P=c_{s}^{2} \varepsilon$, known as the equation of state. Its magnitude depends on the nature of the equation of state, whether it is soft or hard. Therefore, the $c_{s}^{2}$ is calculated from the ratio of the partial derivatives of the pressure and the energy density with respect to the temperature,

$$
C_{s}^{2}=\frac{\partial P}{\partial \varepsilon}=\frac{\partial P / \partial T}{\partial \varepsilon / \partial T}
$$




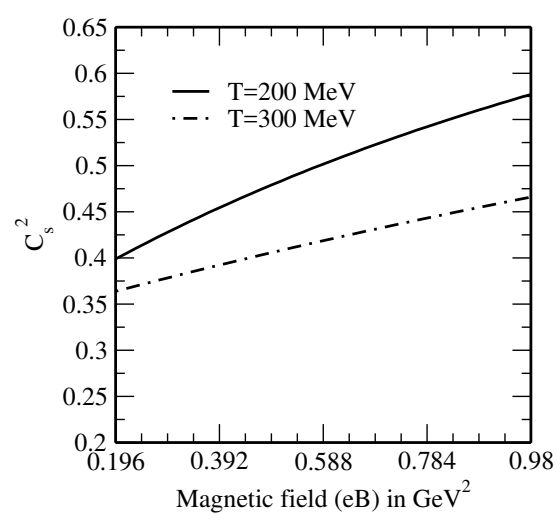

(a)

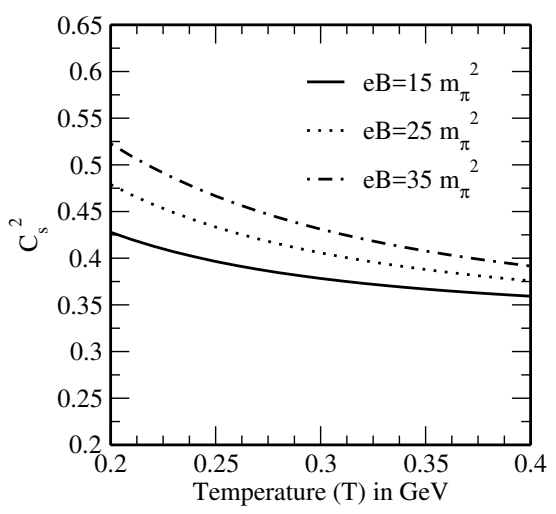

(b)

Figure 7. The variation of square of the speed of sound with strong magnetic field at various temperatures (a) and the variation with temperature in the presence of different strong magnetic fields (b).

As we have observed that the thermodynamic observables are affected by the presence of the magnetic field, so we expect that the equation of state, hence $c_{s}^{2}$ may also be affected by the presence of a strong magnetic field. Therefore we have computed the square of the speed of sound of a hot QCD medium as a function of the external magnetic field in figure 7a. It is found that the speed of sound at a fixed temperature increases with the magnetic fields. This can be understood by the fact that, as the strength of the magnetic field increases, the energy density decreases and the pressure increases, hence the speed of sound increases. From the original perspective of how the EoS of a thermal QCD medium is modified in a magnetic field, we have computed $c_{s}^{2}$ as a function of temperature at different strengths of the magnetic fields in figure $7 \mathrm{~b}$. We found that $c_{s}^{2}$ decreases with the temperature and approaches asymptotically to the ideal value $1 / 3$.

However, when we plot the speed of sound in terms of its ideal limit with the magnetic field at a fixed temperature and vice versa in figures $8 \mathrm{a}$ and $8 \mathrm{~b}$, respectively, we have noticed a dip in the variation of $c_{s}^{2}$ for some specific values of the temperature and the magnetic field. The interesting finding could have the phenomenological implications in the ultrarelativistic heavy ion collisions, because the speed of sound modulates the hydrodynamic expansion of the medium produced in non-central ultra-relativistic heavy ion collisions.

Although it may not be plausible to compare our results on the QCD EoS with the effective model calculations at the equal base, we may however update the effects of the magnetic field on the EoS for the strongly interacting matter in Nambu-Jona-Lasinio (NJL), quark-meson (QM), hadron resonance gas (HRG), MIT bag models etc. For example, in the NJL model framework, the EoS of $\beta$-equilibrium quark matter in a magnetic field becomes stiffer [66], where the light flavors are affected most by the magnetic field [67], as a result the size and the location of the first-order phase transition line get shifted [68]. Similarly in the QM model framework, the presence of a strong magnetic field makes the EoS for neutron star harder than the free-field case [69], as a result the chiral phase transition which was earlier a crossover can be turned into a first-order [70] unlike the NJL model where a crossover remains a crossover [71]. Similarly the HRG models study the 


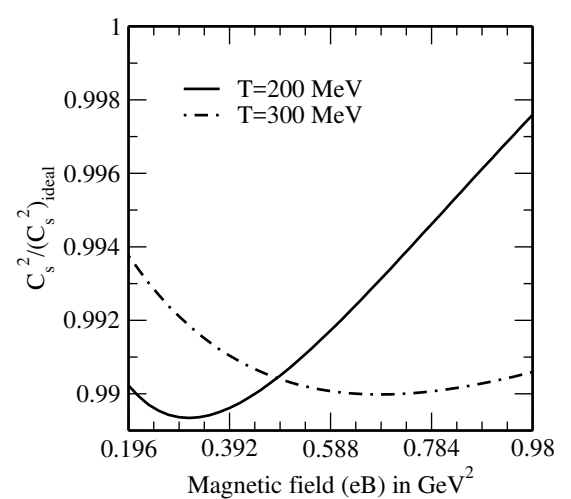

(a)

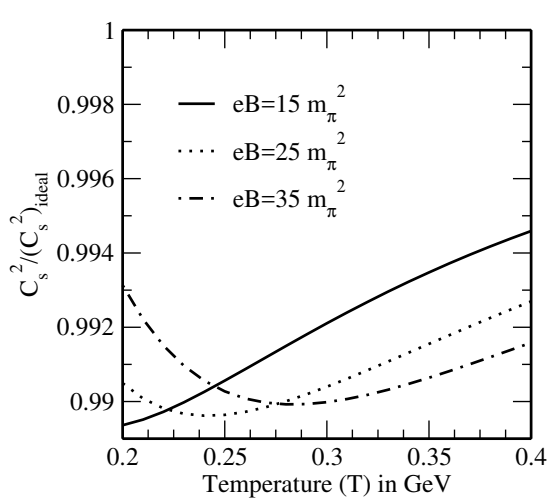

(b)

Figure 8. The variation of $c_{s}^{2}$ normalized by its ideal value as a function of magnetic field at various temperatures (a) and as a function of temperature in the presence of varying strong magnetic fields (b).

thermodynamics of low temperature hadronic phase in the presence of a magnetic field [72] where the pressure, energy density and entropy density are found to increase with the magnetic field. Interestingly the thermodynamic pressure for the QGP phase calculated in the MIT bag model [73] increases with the strong magnetic field and agrees with our one-loop calculation, however the pressure calculated for the hadronic phase cannot be compared with our results.

\section{Conclusions}

In this work, we have explored how the thermodynamic observables of a hot QCD medium in one-loop have been affected in the presence of a very strong magnetic field, which may be produced in the non-central events of ultra-relativistic heavy ion collisions. All thermodynamic observables have contributions from both the quarks and gluons through their respective one-loop self-energies, where the quark contribution has been affected strongly by the magnetic field whereas, the gluon part is largely unaffected except for the softening of the screening mass. As a result, even the pressure for the noninteracting quarks in a strongly magnetized thermal medium gets enhanced and an overall increase in the total pressure of the QCD matter is observed compared to a thermal medium in the absence of an external magnetic field. As a consequence, the entropy density becomes decreased and the energy density too decreases with respect to a pure thermal medium. Finally we obtained the equation of state by calculating the speed of sound of the QCD medium, which is found to increase with the magnetic field and shows a dip when it is plotted in units of its ideal limit in a specific range of the temperatures and the magnetic fields. The above observations could have interesting implications on the expansion dynamics of the medium produced at RHIC and LHC in the presence of a strong magnetic field, which may influence the outcomes of various signatures. In future we would like to explore the paramagnetic response for the hot QCD medium in a strong magnetic field by determining the magnetization and susceptibility. We expect a squeezing of plasma in a direction perpendicular to the magnetic field, which may affect the elliptic flow $\left(v_{2}\right)$. 


\section{Acknowledgments}

We convey our sincere thanks to Bhaswar Chatterjee and Mujeeb Hasan for their constant help during this work.

Open Access. This article is distributed under the terms of the Creative Commons Attribution License (CC-BY 4.0), which permits any use, distribution and reproduction in any medium, provided the original author(s) and source are credited.

\section{References}

[1] V. Skokov, A.Yu. Illarionov and V. Toneev, Estimate of the magnetic field strength in heavy-ion collisions, Int. J. Mod. Phys. A 24 (2009) 5925 [arXiv:0907.1396] [INSPIRE].

[2] A. Bzdak and V. Skokov, Event-by-event fluctuations of magnetic and electric fields in heavy ion collisions, Phys. Lett. B 710 (2012) 171 [arXiv:1111.1949] [INSPIRE].

[3] P.F. Kolb and R. Rapp, Transverse flow and hadrochemistry in Au+Au collisions at $\sqrt{s_{N N}}=200 \mathrm{GeV}$, Phys. Rev. C 67 (2003) 044903 [hep-ph/0210222] [INSPIRE].

[4] P.F. Kolb and U.W. Heinz, Hydrodynamic description of ultrarelativistic heavy ion collisions, nucl-th/0305084 [INSPIRE].

[5] K. Tuchin, Synchrotron radiation by fast fermions in heavy-ion collisions, Phys. Rev. C 82 (2010) 034904 [Erratum ibid. C 83 (2011) 039903] [arXiv: 1006.3051] [INSPIRE].

[6] K. Tuchin, Photon decay in strong magnetic field in heavy-ion collisions, Phys. Rev. C 83 (2011) 017901 [arXiv: 1008.1604] [INSPIRE].

[7] K. Marasinghe and K. Tuchin, Quarkonium dissociation in quark-gluon plasma via ionization in magnetic field, Phys. Rev. C 84 (2011) 044908 [arXiv:1103.1329] [INSPIRE].

[8] K. Fukushima and J.M. Pawlowski, Magnetic catalysis in hot and dense quark matter and quantum fluctuations, Phys. Rev. D 86 (2012) 076013 [arXiv: 1203.4330] [INSPIRE].

[9] V. Voronyuk, V.D. Toneev, W. Cassing, E.L. Bratkovskaya, V.P. Konchakovski and S.A. Voloshin, (Electro-)Magnetic field evolution in relativistic heavy-ion collisions, Phys. Rev. C 83 (2011) 054911 [arXiv:1103.4239] [INSPIRE].

[10] K. Fukushima, D.E. Kharzeev and H.J. Warringa, The Chiral Magnetic Effect, Phys. Rev. D 78 (2008) 074033 [arXiv:0808.3382] [INSPIRE].

[11] D.E. Kharzeev, L.D. McLerran and H.J. Warringa, The effects of topological charge change in heavy ion collisions: 'Event by event P and CP-violation', Nucl. Phys. A 803 (2008) 227 [arXiv:0711.0950] [INSPIRE].

[12] D.E. Kharzeev, The Chiral Magnetic Effect and Anomaly-Induced Transport, Prog. Part. Nucl. Phys. 75 (2014) 133 [arXiv:1312.3348] [INSPIRE].

[13] V. Braguta, M.N. Chernodub, V.A. Goy, K. Landsteiner, A.V. Molochkov and M.I. Polikarpov, Temperature dependence of the axial magnetic effect in two-color quenched QCD, Phys. Rev. D 89 (2014) 074510 [arXiv:1401.8095] [InSPIRE].

[14] M.N. Chernodub, A. Cortijo, A.G. Grushin, K. Landsteiner and M.A.H. Vozmediano, Condensed matter realization of the axial magnetic effect, Phys. Rev. B 89 (2014) 081407 [arXiv: 1311.0878] [INSPIRE]. 
[15] D.E. Kharzeev and D.T. Son, Testing the chiral magnetic and chiral vortical effects in heavy ion collisions, Phys. Rev. Lett. 106 (2011) 062301 [arXiv: 1010.0038] [INSPIRE].

[16] D.E. Kharzeev, J. Liao, S.A. Voloshin and G. Wang, Chiral magnetic and vortical effects in high-energy nuclear collisions - A status report, Prog. Part. Nucl. Phys. 88 (2016) 1 [arXiv: 1511.04050] [INSPIRE].

[17] V.P. Gusynin, V.A. Miransky and I.A. Shovkovy, Catalysis of dynamical flavor symmetry breaking by a magnetic field in (2+1)-dimensions, Phys. Rev. Lett. 73 (1994) 3499 [Erratum ibid. 76 (1996) 1005] [hep-ph/9405262] [INSPIRE].

[18] D.S. Lee, C.N. Leung and Y.J. Ng, Chiral symmetry breaking in a uniform external magnetic field, Phys. Rev. D 55 (1997) 6504 [hep-th/9701172] [INSPIRE].

[19] V.P. Gusynin and I.A. Shovkovy, Chiral symmetry breaking in QED in a magnetic field at finite temperature, Phys. Rev. D 56 (1997) 5251 [hep-ph/9704394] [INSPIRE].

[20] I.A. Shovkovy, Magnetic Catalysis: A Review, Lect. Notes Phys. 871 (2013) 13 [arXiv: 1207.5081] [INSPIRE].

[21] A. Haber, F. Preis and A. Schmitt, Magnetic catalysis in nuclear matter, Phys. Rev. D 90 (2014) 125036 [arXiv:1409.0425] [INSPIRE].

[22] J.O. Andersen, Chiral perturbation theory in a magnetic background-finite-temperature effects, JHEP 10 (2012) 005 [arXiv: 1205.6978] [INSPIRE].

[23] J.O. Andersen, W.R. Naylor and A. Tranberg, Phase diagram of QCD in a magnetic field, Rev. Mod. Phys. 88 (2016) 025001.

[24] M. Strickland, V. Dexheimer and D.P. Menezes, Bulk Properties of a Fermi Gas in a Magnetic Field, Phys. Rev. D 86 (2012) 125032 [arXiv:1209.3276] [INSPIRE].

[25] S. Fayazbakhsh, S. Sadeghian and N. Sadooghi, Properties of neutral mesons in a hot and magnetized quark matter, Phys. Rev. D 86 (2012) 085042 [arXiv:1206.6051] [INSPIRE].

[26] S. Fayazbakhsh and N. Sadooghi, Weak decay constant of neutral pions in a hot and magnetized quark matter, Phys. Rev. D 88 (2013) 065030 [arXiv:1306.2098] [inSPIRE].

[27] G. Basar, D. Kharzeev, D. Kharzeev and V. Skokov, Conformal anomaly as a source of soft photons in heavy ion collisions, Phys. Rev. Lett. 109 (2012) 202303 [arXiv:1206.1334] [INSPIRE].

[28] A. Ayala, J.D. Castano-Yepes, C.A. Dominguez and L.A. Hernandez, Thermal photons from gluon fusion with magnetic fields, EPJ Web Conf. 141 (2017) 02007 [arXiv:1604.02713] [INSPIRE].

[29] N. Sadooghi and F. Taghinavaz, Magnetized plasminos in cold and hot QED plasmas, Phys. Rev. D 92 (2015) 025006 [arXiv: 1504.04268] [INSPIRE].

[30] K. Tuchin, Electromagnetic radiation by quark-gluon plasma in a magnetic field, Phys. Rev. C 87 (2013) 024912 [arXiv:1206.0485] [INSPIRE].

[31] K. Tuchin, Magnetic contribution to dilepton production in heavy-ion collisions, Phys. Rev. C 88 (2013) 024910 [arXiv:1305.0545] [INSPIRE].

[32] K. Tuchin, Particle production in strong electromagnetic fields in relativistic heavy-ion collisions, Adv. High Energy Phys. 2013 (2013) 490495. 
[33] A. Bandyopadhyay, C.A. Islam and M.G. Mustafa, Electromagnetic spectral properties and Debye screening of a strongly magnetized hot medium, Phys. Rev. D 94 (2016) 114034 [arXiv: 1602.06769] [INSPIRE].

[34] N. Sadooghi and F. Taghinavaz, Dilepton production rate in a hot and magnetized quark-gluon plasma, Annals Phys. 376 (2017) 218 [arXiv: 1601.04887] [INSPIRE].

[35] K.A. Mamo, Enhanced thermal photon and dilepton production in strongly coupled $N=4$ SYM plasma in strong magnetic field, JHEP 08 (2013) 083 [arXiv:1210.7428] [INSPIRE].

[36] G.S. Bali, F. Bruckmann, G. Endrödi, S.D. Katz and A. Schäfer, The QCD equation of state in background magnetic fields, JHEP 08 (2014) 177 [arXiv: 1406.0269] [INSPIRE].

[37] N.O. Agasian and S.M. Fedorov, Quark-hadron phase transition in a magnetic field, Phys. Lett. B 663 (2008) 445 [arXiv:0803.3156] [INSPIRE].

[38] G.S. Bali et al., The QCD phase diagram for external magnetic fields, JHEP 02 (2012) 044 [arXiv:1111.4956] [INSPIRE].

[39] G. Endrodi, Critical point in the QCD phase diagram for extremely strong background magnetic fields, JHEP 07 (2015) 173 [arXiv:1504.08280] [INSPIRE].

[40] A. Ayala, M. Loewe and R. Zamora, Inverse magnetic catalysis in the linear $\sigma$-model with quarks, Phys. Rev. D 91 (2015) 016002 [arXiv:1406.7408] [InSPIRE].

[41] A. Ayala, C.A. Dominguez, L.A. Hernández, M. Loewe and R. Zamora, Magnetized effective QCD phase diagram, Phys. Rev. D 92 (2015) 096011 [arXiv:1509.03345] [INSPIRE].

[42] A. Ayala, M. Loewe and R. Zamora, Inverse magnetic catalysis in the linear $\sigma$-model, J. Phys. Conf. Ser. 720 (2016) 012026 [InSPIRE].

[43] A.N. Tawfik, A.M. Diab, N. Ezzelarab and A.G. Shalaby, QCD thermodynamics and magnetization in nonzero magnetic field, Adv. High Energy Phys. 2016 (2016) 1381479.

[44] P.B. Arnold and C.-X. Zhai, The three loop free energy for pure gauge QCD, Phys. Rev. D 50 (1994) 7603 [hep-ph/9408276] [INSPIRE].

[45] P.B. Arnold and C.-x. Zhai, The three loop free energy for high temperature QED and QCD with fermions, Phys. Rev. D 51 (1995) 1906 [hep-ph/9410360] [INSPIRE].

[46] C.-x. Zhai and B.M. Kastening, The Free energy of hot gauge theories with fermions through $g^{* *} 5$, Phys. Rev. D 52 (1995) 7232 [hep-ph/9507380] [inSPIRE].

[47] K. Kajantie, M. Laine, K. Rummukainen and Y. Schröder, The pressure of hot QCD up to $g^{6} \ln (1 / g)$, Phys. Rev. D 67 (2003) 105008 [hep-ph/0211321] [INSPIRE].

[48] E. Braaten and A. Nieto, Free energy of QCD at high temperature, Phys. Rev. D 53 (1996) 3421 [hep-ph/9510408] [INSPIRE].

[49] J.O. Andersen, E. Braaten and M. Strickland, Hard thermal loop resummation of the thermodynamics of a hot gluon plasma, Phys. Rev. D 61 (2000) 014017 [hep-ph/9905337] [INSPIRE].

[50] J.O. Andersen, E. Braaten and M. Strickland, Hard thermal loop resummation of the free energy of a hot quark-gluon plasma, Phys. Rev. D 61 (2000) 074016 [hep-ph/9908323] [INSPIRE].

[51] J.O. Andersen, E. Braaten, E. Petitgirard and M. Strickland, HTL perturbation theory to two loops, Phys. Rev. D 66 (2002) 085016 [hep-ph/0205085] [INSPIRE]. 
[52] J.O. Andersen, E. Petitgirard and M. Strickland, Two loop HTL thermodynamics with quarks, Phys. Rev. D 70 (2004) 045001 [hep-ph/0302069] [INSPIRE].

[53] A. Bandyopadhyay, N. Haque and M.G. Mustafa, The pressure of a weakly magnetized deconfined QCD matter within one-loop Hard-Thermal-Loop perturbation theory, arXiv: 1702.02875 [INSPIRE].

[54] J.S. Schwinger, On gauge invariance and vacuum polarization, Phys. Rev. 82 (1951) 664 [INSPIRE].

[55] W.-y. Tsai, Vacuum Polarization in Homogeneous Magnetic Fields, Phys. Rev. D 10 (1974) 2699 [INSPIRE].

[56] E.J. Ferrer, V. de la Incera and X.J. Wen, Quark Antiscreening at Strong Magnetic Field and Inverse Magnetic Catalysis, Phys. Rev. D 91 (2015) 054006 [arXiv:1407.3503] [INSPIRE].

[57] Yu.A. Simonov, Perturbative theory in the nonperturbative QCD vacuum, Phys. Atom. Nucl. 58 (1995) 107 [hep-ph/9311247] [INSPIRE].

[58] M.A. Andreichikov, V.D. Orlovsky and Yu.A. Simonov, Asymptotic Freedom in Strong Magnetic Fields, Phys. Rev. Lett. 110 (2013) 162002 [arXiv:1211.6568] [inSPIRE].

[59] A. Ayala, J.J. Cobos-Martínez, M. Loewe, M.E. Tejeda-Yeomans and R. Zamora, Finite temperature quark-gluon vertex with a magnetic field in the Hard Thermal Loop approximation, Phys. Rev. D 91 (2015) 016007 [arXiv:1410.6388] [INSPIRE].

[60] A. Chodos, K. Everding and D.A. Owen, QED With a Chemical Potential: 1. The Case of a Constant Magnetic Field, Phys. Rev. D 42 (1990) 2881 [INSPIRE].

[61] N. Sadooghi and K.S. Anaraki, Improved ring potential of QED at finite temperature and in the presence of weak and strong magnetic field, Phys. Rev. D 78 (2008) 125019 [arXiv: 0805.0078] [INSPIRE].

[62] V.P. Gusynin and A.V. Smilga, Electron selfenergy in strong magnetic field: Summation of double logarithmic terms, Phys. Lett. B 450 (1999) 267 [hep-ph/9807486] [INSPIRE].

[63] L. Dolan and R. Jackiw, Symmetry Behavior at Finite Temperature, Phys. Rev. D 9 (1974) 3320 [INSPIRE].

[64] M. Hasan, B. Chatterjee and B.K. Patra, Heavy Quark Potential in a static and strong homogeneous magnetic field, Eur. Phys. J. C 77 (2017) 767 [arXiv:1703.10508] [InSPIRE].

[65] K. Fukushima, K. Hattori, H.-U. Yee and Y. Yin, Heavy Quark Diffusion in Strong Magnetic Fields at Weak Coupling and Implications for Elliptic Flow, Phys. Rev. D 93 (2016) 074028 [arXiv: 1512.03689] [INSPIRE].

[66] D.P. Menezes, M. Benghi Pinto, S.S. Avancini and C. Providencia, Quark matter under strong magnetic fields in the SU(3) Nambu-Jona-Lasinio Model, Phys. Rev. C 80 (2009) 065805 [arXiv: 0907.2607] [INSPIRE].

[67] S.S. Avancini, D.P. Menezes and C. Providencia, Finite temperature quark matter under strong magnetic fields, Phys. Rev. C 83 (2011) 065805 [INSPIRE].

[68] G.N. Ferrari, A.F. Garcia and M.B. Pinto, Chiral Transition Within Effective Quark Models Under Magnetic Fields, Phys. Rev. D 86 (2012) 096005 [arXiv:1207.3714] [InSPIRE].

[69] P. Yue and H. Shen, Quark-meson coupling model for antikaon condensation in neutron star matter with strong magnetic fields, Phys. Rev. C 77 (2008) 045804 [arXiv:0804.3027] [INSPIRE]. 
[70] E.S. Fraga and A.J. Mizher, Chiral transition in a strong magnetic background, Phys. Rev. D 78 (2008) 025016 [arXiv: 0804.1452] [INSPIRE].

[71] J.K. Boomsma and D. Boer, The Influence of strong magnetic fields and instantons on the phase structure of the two-flavor NJLS model, Phys. Rev. D 81 (2010) 074005 [arXiv:0911.2164] [INSPIRE].

[72] G. Endrödi, QCD equation of state at nonzero magnetic fields in the Hadron Resonance Gas model, JHEP 04 (2013) 023 [arXiv:1301.1307] [INSPIRE].

[73] E.S. Fraga and L.F. Palhares, Deconfinement in the presence of a strong magnetic background: an exercise within the MIT bag model, Phys. Rev. D 86 (2012) 016008 [arXiv: 1201.5881] [INSPIRE]. 